

\title{
Comonotonic Approximations for Optimal Portfolio Selection Problems
}

\author{
J. Dhaene \\ S. Vanduffel \\ M. J. Goovaerts \\ R. Kaas \\ D. Vyncke
}

March 28, 2004

\begin{abstract}
We investigate multiperiod portfolio selection problems in a Black \& Scholes type market where a basket of 1 riskless and $m$ risky securities are traded continuously. We look for the optimal allocation of wealth within the class of 'constant mix' portfolios. First, we consider the portfolio selection problem of a decision maker who invests money at predetermined points in time in order to obtain a target capital at the end of the time period under consideration. A second problem concerns a decision maker who invests some amount of money (the initial wealth or provision) in order to be able to fullfil a series of future consumptions or payment obligations. Several optimality criteria and their interpretation within Yaari's dual theory of choice under risk are presented. For both selection problems, we propose accurate approximations based on the concept of comonotonicity, as studied in Dhaene, Denuit, Goovaerts, Kaas \& Vyncke (2002 a,b). Our analytical approach avoids simulation, and hence reduces the computing effort drastically.
\end{abstract}

\section{Introduction}

Strategic portfolio selection is the process used to identify the best allocation of wealth among a basket of securities for an investor with a given consumption/saving behavior over a given investment horizon. The basket of available securities will typically be a selection of risky assets such as stocks, bonds and real estate, and risk-free components such as cash and money market instruments. The individual investor or the asset manager chooses an initial asset mix and a particular tactical trading strategy within a given set of strategies, according to which he will buy and sell risky and risk-free assets, during the whole time period under consideration.

The simplest case is a static strategy called 'buy and hold': the investments are performed according to a given strategy and no rebalancing is performed during the investment period. Single-index benchmarking, e.g. replicating a single stock market index, is a buy-and-hold strategy in case the index weights are not changed over the investment period.

Other strategies are called dynamic in the sense that they imply a periodic rebalancing process of the assets. A 'constant mix' strategy implies keeping the initial proportions constant, as opposed to a 'buy and hold' strategy where the initial quantities are kept 
constant through time. As the prices of assets evolve randomly over time, a constant mix strategy requires buying and/or selling at each time instant in order to keep the fractions at the predetermined level. Such a strategy implies a 'buy low and sell high' rule in the sense that price and asset-purchase are counter-varying: if the price of a single asset goes up while the prices of the other assets remain constant, then the quantity of the single asset should be decreased and vice versa. Furthermore, if the stock market has decreased, one has to buy stocks against the riskfree component, whereas if the stock market has increased one has to sell stock and buy riskfree instruments. Multiple-index benchmarking, e.g. replicating a benchmark which consists of the average of a stock benchmark and a bond benchmark, implies a constant mix strategy in order to stick to the benchmark proportions.

In this paper we will investigate multi-period optimal portfolio selection problems in a Black \& Scholes (1973) lognormal setting. We will assume that the investor has to choose the optimal investment strategy for a given consumption or savings pattern, within the class of constant mix strategies. We will consider two general types of problems, which will be referred to as the terminal wealth problem and the reserving problem respectively.

In the terminal wealth problem, the decision maker will invest a given series of positive saving amounts $\alpha_{0}, \alpha_{1}, \ldots, \alpha_{n-1}$ at predetermined times $0,1, \ldots, n-1$ such that his terminal wealth at time $n$ will reach or exceed some target capital $K$ with a sufficiently large probability.

As terminal wealth is a sum of dependent lognormal random variables, its distribution function cannot be determined exactly and is too cumbersome to work with. Therefore, we will present accurate approximations for the distribution function at hand. The first approximation that we will consider for the distribution of terminal wealth will be called the 'comonotonic upper bound' as it is an upper bound for the exact distribution in the convex order sense. It is derived by keeping the marginal distributions exact but approximating the copula that describes the dependency structure between the random accumulation factors involved by the comonotonic copula.

Our second approximation for the exact distribution is based on the technique of conditioning. In this approach, the marginal distributions are changed and as a result the copula describing the dependency structure is replaced by the comonotonic copula. We will call this the 'comonotonic lower bound' approach as it can be proven that it is a lower bound in the sense of convex ordering. Especially this lower bound will perform very accurately as an approximation to the exact distribution.

The approximations that we propose have several advantages. First, for any given investment strategy they provide an accurate and easy to compute approximation for any risk measure that is additive for comonotonic risks, such as distortion risk measures (VaR and TailVaR for instance). Second, it turns out that for the comonotonic approximations we propose, the optimal investment mix can be found on the mean-variance efficient frontier. Third, the comonotonic approximations reduce the multivariate randomness of the multiperiod problem to a univariate randomness.

The optimal investment mix could be defined as the one that requires the smallest constant amount $\alpha$ that has to be invested from period to period in order to reach a final wealth of at least $K$ with a probability of at least $1-\epsilon$. Or, one could define the optimal mix as the one that maximizes the probability of reaching terminal wealth of at least $K$ for a given investment of $\alpha$ per month. 
The proposed methodology can be used to solve several personal finance problems. A first problem is what one could call the 'saving for retirement problem'. In this case, one wants to retire in $n$ years with a 'nest egg' of $K$ - in real terms, i.e. in today's Euro's. How much does one have to save monthly - in real terms - in order to assure a $(1-\epsilon)$ chance to reach the retirement financial goal? Clearly the answer will depend on the investment mix. The theory on comonotonicity gives a quick, elegant and accurate answer to this question.

A second personal finance problem where the methodology can be used is the situation where an individual underwrites an $n$ year loan of $K$ at a yearly interest rate of $i$. The first way to pay back the loan is by a classical annuity where the lender pays at the end of each of the coming $n$ years a fixed amount of $\frac{K}{a_{\bar{n} \mid i}}$. From the viewpoint of the lender, an annuity loan is equivalent with a loan with yearly interest payments of $K i$ while a yearly amount of $\frac{K}{a_{\bar{n} \mid i}}-K i$ is invested in an amortization fund with a fixed return of $i$. At the end of the $n$ year period the amortization fund will have grown to the required amount of $K$. An alternative is to pay the yearly interest of $K i$ and in addition, invest a yearly amount $\alpha$ in an amortization fund with future stochastic returns. The amount $\alpha$ is chosen such that the probability that the value of the amortization fund at time $n$ will exceed the amount $K$ will be sufficiently large.

In the reserving problem, which is in some sense dual to the final wealth problem, the decision maker targets a given series of future consumptions $\alpha_{1}, \alpha_{1}, \ldots, \alpha_{n}$ at times $1,2, \ldots, n$. He sets up an initial reserve $R_{0}$ and wants to invest this amount in such a way that the possibility of reaching his targets is maximized.

For this type of investment problems, the optimal investment mix could be defined as the one that leads to the largest survival probability, given the initial reserve. Or, one could fix the required survival probability and determine the optimal investment strategy as the one that minimizes the required initial reserve.

One possible application is the problem of the decision maker who is faced with a series of deterministic obligations or liabilities due at predetermined fixed points in time. He wants to determine the reserve (and/or total balance sheet capital requirement) and selects the optimal investment portfolio such that the possibility of ruin is minimized.

An application in the area of personal finance is the annuitization problem where an initial amount $R_{0}$ is invested and used to enable a series of future periodic payments. A somewhat related problem is the so-called 'after-retirement problem'. The difference with our setting is that in the after-retirement problem the time-horizon is random and equal to the remaining life time. This problem is considered in Milevsky, Ho \& Robinson (1997) and Milevsky \& Robinson (2000). These authors take the investment strategy as given and find the corresponding probability of lifetime ruin. Young (2004) on the other hand finds the optimal dynamic investment strategy as the one that minimizes the probability of lifetime ruin.

As the time horizon that we consider is long (typically 10, 20 or more years), assuming a Gaussian model seems to be appropriate, at least approximately, by the Central Limit Theorem. In order to verify whether the theoretical setup can be approximately compared with the data generating mechanism of real situations, we refer to Cesari \& Cremonini (2003). They investigate four well-known stock market indices in US dollars, from Morgan Stanley: MSCI World, North America, Europe and Pacific, covering all major stock markets in industrial as well as emerging countries. For the period 1997-1999, the authors conclude 
that weekly (and longer period) returns can be considered as normal and independent. Daily returns on the other hand are both non-normal and autocorrelated.

The paper is organized as follows. In Sections 2 and 3 we present some results concerning risk measures, comonotonicity, the Black \& Scholes setting, constant mix portfolios and mean-variance analysis that will be used throughout the paper. Next, the problem of finding optimal investment strategies in a general multivariate final wealth model with savings at discrete points in time is analyzed in Section 4. The dual problem of setting an initial provision and optimizing investments in a general model with consumptions at discrete points in time is considered in Section 5. Final conclusions and some possible generalizations are discussed in Section 6.

To the best of our knowledge, determining optimal investment strategies for terminal wealth and optimal provision problems by means of the comonotonic approach, as presented in Sections 4 and 5, is new. The research was motivated by our practical experience concerning optimal portfolio selection problems of some banking and insurance institutions in Belgium.

\section{Risk measures and comonotonicity}

In this section, we will introduce some definitions and present some results related to risk measures and comonotonicity that will be used throughout this paper. More details about comonotonicity can be found in Dhaene, Denuit, Goovaerts, Kaas \& Vyncke (2002a,b), more details about the relation between risk measures and comonotonicity can be found in Dhaene, Vanduffel, Tang, Goovaerts, Kaas \& Vyncke (2004).

\subsection{Risk measures}

A risk measure summarizes the information contained in the distribution function of a random variable in one single real number. For a random variable $X$, the $p$-quantile risk measure is defined as

$$
Q_{p}[X]=\inf \left\{x \in \mathbb{R} \mid F_{X}(x) \geq p\right\}, \quad p \in(0,1),
$$

where $F_{X}(x)=\operatorname{Pr}[X \leq x]$ and by convention, $\inf \{\phi\}=+\infty$. A related risk measure is denoted by $Q_{p}^{+}[X]$ and is defined by

$$
Q_{p}^{+}[X]=\sup \left\{x \in \mathbb{R} \mid F_{X}(x) \leq p\right\}, \quad p \in(0,1),
$$

where by convention $\sup \{\phi\}=-\infty$. Note that only values of $p$ corresponding to a horizontal segment of $F_{X}$ lead to different values of $Q_{p}[X]$ and $Q_{p}^{+}[X]$. Hence, if $F_{X}$ is strictly increasing, both risk measures will coincide for all values of $p$. In this case, we can also define the $(1-p)$-quantiles by

$$
Q_{1-p}[X]=\sup \left\{x \in \mathbb{R} \mid \bar{F}_{X}(x) \geq p\right\}, \quad p \in(0,1),
$$

where $\bar{F}_{X}(x)=1-F_{X}(x)$.

In the sequel, we will always consider random variables with finite mean. The Conditional Tail Expectation (CTE) at level $p$ will be denoted by $\mathrm{CTE}_{p}[X]$. It is defined by

$$
C T E_{p}[X]=E\left[X \mid X>Q_{p}[X]\right], \quad p \in(0,1) .
$$


The CTE measures the right tail of the distribution function. We will also need a risk measure that measures the left tail of the distribution function. Therefore, we introduce the Conditional Left Tail Expectation, which is defined by

$$
\operatorname{CLTE}_{p}[X]=E\left[X \mid X<Q_{p}^{+}[X]\right] .
$$

One can prove that the following relation holds between CTE and CLTE:

$$
C L T E_{1-p}[X]=-C T E_{p}[-X], \quad p \in(0,1) .
$$

\subsection{Comonotonic bounds for sums of dependent random variables}

A random vector $\underline{Y}=\left(Y_{0}, Y_{1}, \cdots, Y_{n}\right)$ is said to be comonotonic if

$$
\left(Y_{0}, Y_{1}, \cdots, Y_{n}\right) \stackrel{d}{=}\left(F_{Y_{0}}^{-1}(U), F_{Y_{1}}^{-1}(U), \cdots, F_{Y_{n}}^{-1}(U)\right)
$$

where $U$ is a random variable which is uniformly distributed on the unit interval and where the notation $\stackrel{d}{=}$ stands for 'equality in distribution'.

For any random vector $\underline{X}=\left(X_{0}, X_{1}, \cdots, X_{n}\right)$, we will call its comonotonic counterpart any random vector with the same marginal distributions and with the comonotonic dependency structure. The comonotonic counterpart of $\underline{X}=\left(X_{0}, X_{1}, \cdots, X_{n}\right)$ will be denoted by $\underline{X}^{c}=$ $\left(X_{0}^{c}, X_{1}^{c}, \cdots, X_{n}^{c}\right)$. Hence for any random vector $\underline{X}=\left(X_{0}, X_{1}, \cdots, X_{n}\right)$, we have

$$
\left(X_{0}^{c}, X_{1}^{c}, \cdots, X_{n}^{c}\right) \stackrel{d}{=}\left(F_{X_{0}}^{-1}(U), F_{X_{1}}^{-1}(U), \cdots, F_{X_{n}}^{-1}(U)\right)
$$

It can be proven that a random vector is comonotonic if and only if all its marginals are non-decreasing functions (or all are non-increasing functions) of the same random variable.

The random variable $X$ is said to precede the random variable $Y$ in the stop-loss order sense, notation $X \leq_{s l} Y$, if $X$ has lower stop-loss premiums than $Y$ :

$$
\mathrm{E}\left[(X-d)_{+}\right] \leq \mathrm{E}\left[(Y-d)_{+}\right], \quad-\infty<d<+\infty .
$$

On the other hand, $X$ is said to precede $Y$ in the convex order sense, notation $X \leq_{c x} Y$, if $X \leq_{s l} Y$ and in addition $\mathrm{E}[X]=\mathrm{E}[Y]$. An introduction to ordering of (distributions of) random variables, with actuarial applications, can be found in Chapter 10 of Kaas, Goovaerts, Dhaene \& Denuit (2001).

Theorem 2.1 (Convex bounds for sums of random variables). For any random vector $\left(X_{0}, X_{1}, \cdots, X_{n}\right)$ and any random variable $\Lambda$, we have that

$$
\sum_{i=0}^{n} E\left[X_{i} \mid \Lambda\right] \leq_{c x} \sum_{i=0}^{n} X_{i} \leq_{c x} \sum_{i=0}^{n} F_{X_{i}}^{-1}(U) .
$$

The theorem above states that the least attractive random vector $\left(X_{0}, X_{1}, \cdots, X_{n}\right)$ with given marginals $F_{X_{i}}$, in the sense that the sum of its components is largest in the convex order, has the comonotonic joint distribution, which means that it has the joint distribution of $\left(F_{X_{0}}^{-1}(U), F_{X_{1}}^{-1}(U), \cdots, F_{X_{n}}^{-1}(U)\right)$. The components of this random vector are maximally 
dependent, all components being non-decreasing functions of the same random variable.

The random variable $S^{c}=\sum_{i=0}^{n} F_{X_{i}}^{-1}(U)$ will be called the comonotonic upper bound of $S=\sum_{i=0}^{n} X_{i}$, whereas the random variable $S^{l}=\sum_{i=0}^{n} E\left[X_{i} \mid \Lambda\right]$ will be referred to as a lower bound for $S$.

The random vector $\left(E\left[X_{0} \mid \Lambda\right], E\left[X_{1} \mid \Lambda\right], \cdots, E\left[X_{n} \mid \Lambda\right]\right)$ will in general not have the same marginal distributions as $\left(X_{0}, X_{1}, \cdots, X_{n}\right)$. If one can find a conditioning random variable $\Lambda$ with the property that all random variables $E\left[X_{i} \mid \Lambda\right]$ are non-increasing functions of $\Lambda$ (or all are non-decreasing functions of $\Lambda$ ), then the lower bound $S^{l}=\sum_{i=0}^{n} E\left[X_{i} \mid \Lambda\right]$ is a sum of $n$ comonotonic random variables. In the sequel, we will often use a comonotonic approximation for a sum of non-independent random variables. The advantage of the comonotonic dependency structure is that any distortion risk measure of a sum of comonotonic random variables equals the sum of the risk measures of the marginals involved. For the quantile risk measures defined above, we find for all $p \in(0,1)$ :

$$
\begin{aligned}
Q_{p}\left[S^{c}\right] & =\sum_{i=0}^{n} Q_{p}\left[X_{i}\right], \\
Q_{p}^{+}\left[S^{c}\right] & =\sum_{i=0}^{n} Q_{p}^{+}\left[X_{i}\right] .
\end{aligned}
$$

For the CTE and the CLTE a similar result can be proven, provided all marginal distributions $F_{X_{i}}$ are continuous:

$$
\begin{aligned}
C T E_{p}\left[S^{c}\right] & =\sum_{i=0}^{n} C T E_{p}\left[X_{i}\right], \text { provided all } F_{X_{i}} \text { are continuous, } \\
C L T E_{p}\left[S^{c}\right] & =\sum_{i=0}^{n} C L T E_{p}\left[X_{i}\right], \text { provided all } F_{X_{i}} \text { are continuous. }
\end{aligned}
$$

\subsection{Sums of lognormal random variables}

Consider the sum

$$
S=\sum_{i=0}^{n} \alpha_{i} e^{Z_{i}}
$$

where the $\alpha_{i}$ are non-negative constants and the $Z_{i}$ are linear combinations of the components of the random vector $\left(Y_{1}, Y_{2}, \cdots, Y_{n}\right)$ which is assumed to have a multivariate normal distribution:

$$
Z_{i}=\sum_{j=1}^{n} \lambda_{i j} Y_{j}
$$

Let $U$ be uniformly distributed on the unit interval. The comonotonic upper bound $S^{c}=$ $\sum_{i=0}^{n} F_{\alpha_{i} e^{z_{i}}}^{-1}(U)$ of $S$ is given by

$$
S^{c}=\sum_{i=0}^{n} \alpha_{i} e^{E\left[Z_{i}\right]+\sigma_{Z_{i}} \Phi^{-1}(U)} .
$$


Taking into account the additivity property, the following expressions can be derived for the risk measures associated with $S^{c}$ :

$$
\begin{aligned}
Q_{p}\left[S^{c}\right] & =Q_{p}^{+}\left[S^{c}\right]=\sum_{i=0}^{n} \alpha_{i} e^{E\left[Z_{i}\right]+\sigma_{Z_{i}} \Phi^{-1}(p)} \\
C T E_{p}\left[S^{c}\right] & =\sum_{i=0}^{n} \alpha_{i} e^{E\left[Z_{i}\right]+\frac{1}{2} \sigma_{Z_{i}}^{2}} \frac{\Phi\left(\sigma_{Z_{i}}-\Phi^{-1}(p)\right)}{1-p}, \\
C L T E_{p}\left[S^{c}\right] & =\sum_{i=0}^{n} \alpha_{i} e^{E\left[Z_{i}\right]+\frac{1}{2} \sigma_{Z_{i}}^{2}} \frac{1-\Phi\left(\sigma_{Z_{i}}-\Phi^{-1}(p)\right)}{p}, \quad p \in(0,1) .
\end{aligned}
$$

In order to define a comonotonic lower bound $S^{l}$ for $S$, we choose a conditioning random variable $\Lambda$ which is a linear combination of the $Y_{j}$ :

$$
\Lambda=\sum_{j=1}^{n} \beta_{j} Y_{j}
$$

After some computations, we find that the lower bound $S^{l}=\sum_{i=0}^{n} \alpha_{i} E\left[e^{Z_{i}} \mid \Lambda\right]$ is given by

$$
S^{l}=\sum_{i=0}^{n} \alpha_{i} e^{E\left[Z_{i}\right]+\frac{1}{2}\left(1-r_{i}^{2}\right) \sigma_{Z_{i}}^{2}+r_{i} \sigma_{Z_{i}} \Phi^{-1}(U)}
$$

where the uniformly distributed random variable $U$ follows from $\Phi^{-1}(U) \equiv \frac{\Lambda-E(\Lambda)}{\sigma_{\Lambda}}$, and $r_{i}$ is the correlation between $Z_{i}$ and $\Lambda$.

If all $r_{i}$ are positive, then $S^{l}$ is a comonotonic sum, which means that quantiles and conditional tail expectations related to $S^{l}$ can be computed by summing the associated risk measures for the marginals involved. Hence, assuming that all $r_{i}$ are positive, we find the following expressions for the risk measures associated with $S^{l}$ :

$$
\begin{aligned}
Q_{p}\left[S^{l}\right] & =Q_{p}^{+}\left[S^{l}\right]=\sum_{i=0}^{n} \alpha_{i} e^{E\left[Z_{i}\right]+\frac{1}{2}\left(1-r_{i}^{2}\right) \sigma_{Z_{i}}^{2}+r_{i} \sigma_{Z_{i}} \Phi^{-1}(p)}, \\
C T E_{p}\left[S^{l}\right] & =\sum_{i=0}^{n} \alpha_{i} e^{E\left[Z_{i}\right]+\frac{1}{2} \sigma_{Z_{i}}^{2}} \frac{\Phi\left(r_{i} \sigma_{Z_{i}}-\Phi^{-1}(p)\right)}{1-p}, \\
C L T E_{p}\left[S^{l}\right] & =\sum_{i=0}^{n} \alpha_{i} e^{E\left[Z_{i}\right]+\frac{1}{2} \sigma_{Z_{i}}^{2}} \frac{1-\Phi\left(r_{i} \sigma_{Z_{i}}-\Phi^{-1}(p)\right)}{p}, \quad p \in(0,1) .
\end{aligned}
$$

The correlation coefficients $r_{i}$ follow from the correlations between the random variables $Y_{i}$. In the special case that all $Y_{i}$ are i.i.d., we find

$$
r_{i}=\frac{\sum_{j=1}^{n}}{\sqrt{\sum_{j=1}^{n} \lambda_{i j}^{2}} \frac{\lambda_{i j} \beta_{j}}{\sqrt{\sum_{j=1}^{n} \beta_{j}^{2}}}}, \quad i=1,2, \cdots, n .
$$

As we have that

$$
\operatorname{Var}[S]=\operatorname{Var}\left[S^{l}\right]+E[\operatorname{Var}[S \mid \Lambda]]
$$


it seems reasonable to choose the coefficients $\beta_{j}$ in (17) such that the variance of $S^{l}$ is maximized. Determining these optimal coefficients $\beta_{j}$ would require tim-consuming numerical calculations. However, we can approximate the optimal coefficients by deriving an approximate expression for $\operatorname{Var}\left[S^{l}\right]$. From Dhaene, Denuit, Kaas, Goovaerts \& Vyncke (2002), we find that $\operatorname{Var}\left[S^{l}\right]$ is given by

$$
\begin{aligned}
\operatorname{Var}\left[S^{l}\right] & =\sum_{i=0}^{n} \sum_{j=0}^{n} \alpha_{i} \alpha_{j} e^{E\left[Z_{i}\right]+E\left[Z_{j}\right]+\frac{1}{2}\left(\sigma_{Z_{i}}^{2}+\sigma_{Z_{j}}^{2}\right)}\left(e^{r_{i} r_{j} \sigma_{Z_{i}} \sigma_{Z_{j}}}-1\right) \\
& \approx \sum_{i=0}^{n} \sum_{j=0}^{n} \alpha_{i} \alpha_{j} e^{E\left[Z_{i}\right]+E\left[Z_{j}\right]+\frac{1}{2}\left(\sigma_{Z_{i}}^{2}+\sigma_{Z_{j}}^{2}\right)} r_{i} r_{j} \sigma_{Z_{i}} \sigma_{Z_{j}} \\
& =\left(\operatorname{corr}\left[\sum_{i=0}^{n} \alpha_{i} e^{E\left[Z_{i}\right]+\frac{1}{2} \sigma_{Z_{i}}^{2}} Z_{i} ; \Lambda\right]\right)^{2} \cdot \operatorname{Var}\left[\sum_{i=0}^{n} \alpha_{i} e^{E\left[Z_{i}\right]+\frac{1}{2} \sigma_{Z_{i}}^{2} Z_{i}}\right] .
\end{aligned}
$$

This approximation will perform well, provided $\sigma_{Z_{i}} \sigma_{Z_{j}}$ is sufficiently small. In the special case that all $Y_{i}$ are i.i.d., this comes down to requiring that the variance of the $Y_{i}$ is small enough.

The approximation for $\operatorname{Var}\left[S^{l}\right]$ is maximized by choosing the correlation coefficient equal to 1 . Hence, by chosing $\Lambda$ equal to

$$
\Lambda=\sum_{i=0}^{n} \alpha_{i} e^{E\left[Z_{i}\right]+\frac{1}{2} \sigma_{Z_{i}}^{2}} Z_{i},
$$

which means that the $\beta_{j}$ - coefficients are given by

$$
\beta_{j}=\sum_{i=0}^{n} \alpha_{i} \lambda_{i j} e^{E\left[Z_{i}\right]+\frac{1}{2} \sigma_{Z_{i}}^{2}}
$$

\section{Stochastic return processes}

\subsection{The Black \& Scholes setting}

Throughout the paper, we will assume the classical continuous-time framework that was pioneered by Merton (1971) and is nowadays mostly referred to as the Black \& Scholes (1973) setting. We suppose that there is a market in which $(m+1)$ securities (assets or investment accounts) are traded continuously. One of the assets is the riskfree asset. Let $P^{0}(0)=P^{0}>0$ be the current price, at time 0 , of 1 unit of the riskfree asset, whereas $P^{0}(t)$ is its price at time $t$. This price is assumed to evolve according to the following ordinary differential equation:

$$
\frac{\mathrm{d} P^{0}(t)}{P^{0}(t)}=r \mathrm{~d} t
$$

with $r>0$. On the other hand, let $P^{i}(0)=P^{i}>0$ be the current price, at time 0 , of 1 unit of risky asset $i$, whereas $P^{i}(t)$ is the price at time $t$ (including reinvestment of dividend income) 
of one unit of risky asset $i$. The price process $P^{i}(t)$ evolves according to a geometric Brownian motion stochastic process, represented by the following stochastic differential equation:

$$
\frac{\mathrm{d} P^{i}(t)}{P^{i}(t)}=\mu_{i} \mathrm{~d} t+\sum_{j=1}^{d} \bar{\sigma}_{i j} \mathrm{~d} W^{j}(t), \quad i=1, \cdots, m,
$$

where $\left(W^{1}(\tau), W^{2}(\tau), \cdots, W^{d}(\tau)\right)$ is a $d$-dimensional standard Brownian motion process. The $W^{i}(\tau)$ are mutually independent standard Brownian motions.

The $m$-dimensional vector $\mu^{T}=\left(\mu_{1} \cdots \mu_{m}\right)$ is called the drift vector of the risky assets. We will assume that $\mu \neq r \mathbf{1}$, with $\mathbf{1}^{T}=\left(\begin{array}{lll}1 & 1 & \ldots\end{array}\right)$.

The $(m \times d)$ matrix $\bar{\Sigma}$ defined by

$$
\bar{\Sigma}=\left(\begin{array}{cccc}
\bar{\sigma}_{11} & \bar{\sigma}_{12} & \cdots & \bar{\sigma}_{1 d} \\
\bar{\sigma}_{21} & \bar{\sigma}_{22} & \cdots & \bar{\sigma}_{2 d} \\
\cdots & \cdots & \cdots & \cdots \\
\bar{\sigma}_{m 1} & \bar{\sigma}_{m 2} & \cdots & \bar{\sigma}_{m d}
\end{array}\right)
$$

is called the diffusion matrix. Further, we define the $(m \times m)$ matrix $\boldsymbol{\Sigma}$ as

$$
\boldsymbol{\Sigma}=\bar{\Sigma} \cdot \overline{\boldsymbol{\Sigma}}^{T}=\left(\begin{array}{cccc}
\sigma_{1}^{2} & \sigma_{12} & \cdots & \sigma_{1 m} \\
\sigma_{21} & \sigma_{2}^{2} & \cdots & \sigma_{2 m} \\
\cdots & \cdots & \cdots & \cdots \\
\sigma_{m 1} & \sigma_{m 2} & \cdots & \sigma_{m}^{2}
\end{array}\right)
$$

with coefficients $\sigma_{i j}$ and $\sigma_{i}^{2}$ given by $\sigma_{i j}=\sum_{k=1}^{d} \bar{\sigma}_{i k} \bar{\sigma}_{j k}$ and $\sigma_{i}^{2}=\sigma_{i i}$. We have that $\sigma_{i j}=$ $\sigma_{j i}$. The matrix $\boldsymbol{\Sigma}$ is called the variance-covariance matrix. We will assume that $\boldsymbol{\Sigma}$ is positive definite. This means that for all non-zero vectors $\pi^{T}=\left(\pi_{1}, \pi_{2}, \cdots, \pi_{m}\right)$ we have that

$$
\pi^{T} \cdot \boldsymbol{\Sigma} \cdot \pi>0
$$

In particular, this assumption implies that all $\sigma_{i}$ are strictly positive. Hence, all $m$ risky assets are indeed risky. It also implies that $\boldsymbol{\Sigma}$ is non-singular, meaning that its determinant is strictly positive, and hence $\boldsymbol{\Sigma}$ has a matrix inverse $\boldsymbol{\Sigma}^{-1}$. As we will see further on, the elements of the matrix $\boldsymbol{\Sigma}$ describe the covariances between the yearly returns of the different investment accounts.

We define the process $B^{i}(\tau)$ by

$$
B^{i}(\tau)=\frac{1}{\sigma_{i}} \sum_{j=1}^{d} \bar{\sigma}_{i j} W^{j}(\tau)
$$

It is straightforward to verify that all $B^{i}(\tau)$ are (correlated) standard Brownian motions, with

$$
\operatorname{Cov}\left(B^{i}(t), B^{j}(t+s)\right)=\frac{\sigma_{i j}}{\sigma_{i} \sigma_{j}} t, \quad t, s \geq 0 .
$$

Rewriting equation (26), we find

$$
\frac{\mathrm{d} P^{i}(t)}{P^{i}(t)}=\mu_{i} \mathrm{~d} t+\sigma_{i} \mathrm{~d} B^{i}(t), \quad i=1, \cdots, m .
$$


The solution to equation (32) is

$$
P^{i}(t)=P^{i} \exp \left\{\left(\mu_{i}-\frac{1}{2} \sigma_{i}^{2}\right) t+\sigma_{i} B^{i}(t)\right\},
$$

which means that $\frac{P^{i}(t)}{P^{i}}$ is lognormally distributed with parameters $\left(\mu_{i}-\frac{1}{2} \sigma_{i}^{2}\right) t$ and $\sigma_{i}^{2} t$, respectively. This implies that the expectation and standard deviation of the price of asset $i$ at time $t$ are given by

$$
\begin{aligned}
& E\left[P^{i}(t)\right]=P^{i} e^{\mu_{i} t}, \\
& \sigma\left[P^{i}(t)\right]=P^{i} e^{\mu_{i} t} \sqrt{e^{\sigma_{i}^{2} t}-1} .
\end{aligned}
$$

Let $k=1,2, \ldots$ Investing an amount of 1 at time $k-1$ in investment account $i$ will grow to the random amount $e^{Y_{k}^{i}}$ at time $k$, where $Y_{k}^{i}$ denotes the yearly return in year $k$ of account $i$. One finds that

$$
Y_{k}^{i}=\left(\mu_{i}-\frac{1}{2} \sigma_{i}^{2}\right)+\sigma_{i}\left(B^{i}(k)-B^{i}(k-1)\right) .
$$

Hence, it follows that the random yearly returns $Y_{k}^{i}$ of asset $i$ are independent and have identical normal distributions with

$$
\begin{aligned}
E\left[Y_{k}^{i}\right] & =\mu_{i}-\frac{1}{2} \sigma_{i}^{2}, \\
\operatorname{Var}\left[Y_{k}^{i}\right] & =\sigma_{i}^{2}, \\
\operatorname{Cov}\left[Y_{k}^{i}, Y_{l}^{j}\right] & =\left\{\begin{array}{ll}
0 & k \neq l \\
\sigma_{i j} & k=l
\end{array} .\right.
\end{aligned}
$$

As stated earlier, the matrix $\boldsymbol{\Sigma}$ is the variance-covariance matrix of the yearly return vector $\left(Y_{k}^{1}, Y_{k}^{2}, \cdots, Y_{k}^{n}\right)$. More details on the translation between the two formalisms (26) and (32) for describing the multivariate asset process can be found e.g. in Björk (1998).

\subsection{Constant mix strategies}

Assume one can invest wealth in one or more of the $m+1$ assets as defined above. Let $\pi(t)^{T}=$ $\left(\pi_{1}(t), \pi_{2}(t), \cdots, \pi_{m}(t)\right)$ be the vector describing the portfolio process, i.e. $\pi_{i}(t)$ is the fraction of the wealth that is invested in risky asset $i$ at time $t$. The residual, i.e. $1-\sum_{i=1}^{n} \pi_{i}(t)$ is invested in the riskfree asset, or, if negative, finances the risky asset purchases. A negative proportion invested in the riskfree asset means borrowing (going short) on the riskfree asset.

We will restrict to constant portfolios $\pi(t)^{T}=\pi^{T}=\left(\pi_{1}, \pi_{2}, \cdots, \pi_{m}\right)$, which means that the fractions invested in the different assets remain constant over time. Investing according to a constant portfolio process implies that one has to follow a dynamic trading strategy. Indeed, as the risky asset returns evolve randomly, one has to trade at each instant in order to keep the fractions invested in the different assets constant. Such investment strategies are known as constant mix strategies, or also as constant proportional investment strategies. Optimality of constant mix strategies in a utility theory setting is considered in Merton (1971). 
Let us now consider one unit of a security that is constructed according to the continuously rebalanced investment strategy $\left(\pi_{1}, \pi_{2}, \cdots, \pi_{m}\right)$, and let $P(t)$ be the price of that unit at time $t$, with $P(0)=P$. One can prove that the price process $P(t)$ evolves according to the dynamics

$$
\begin{aligned}
\frac{\mathrm{d} P(t)}{P(t)} & =\sum_{i=1}^{m} \pi_{i} \frac{\mathrm{d} P^{i}(t)}{P^{i}(t)}+\left(1-\sum_{i=1}^{m} \pi_{i}\right) \frac{\mathrm{d} P^{0}(t)}{P^{0}(t)} \\
& =\left(\sum_{i=1}^{m} \pi_{i}\left(\mu_{i}-r\right)+r\right) \mathrm{d} t+\sum_{i=1}^{m} \pi_{i} \sigma_{i} \mathrm{~d} B^{i}(t) .
\end{aligned}
$$

For a non-zero vector $\pi$, define the process $B(\tau)$ by

$$
B(\tau)=\frac{1}{\sqrt{\pi^{T} \cdot \Sigma \cdot \pi}} \sum_{i=1}^{m} \pi_{i} \sigma_{i} B^{i}(\tau) .
$$

One can verify that $B(\tau)$ is a standard Brownian motion. Equation (37) can then be rewritten as

$$
\frac{\mathrm{d} P(t)}{P(t)}=\mu(\pi) \mathrm{d} t+\sigma(\pi) \mathrm{d} B(t)
$$

with

$$
\mu(\pi)=r+\pi^{T} \cdot(\mu-r \mathbf{1}) \quad \text { and } \quad \sigma^{2}(\pi)=\pi^{T} \cdot \boldsymbol{\Sigma} \cdot \pi,
$$

where $\mathbf{1}$ is the $m$ - vector $(11 \cdots 1)$. Hence, we find that when the portfolio is rebalanced in continuous time in order to keep the fractions constant, the portfolio return is also lognormal distributed. Recall that we assumed that the variance-covariance matrix $\Sigma$ is positive definite. This means that any non-zero combination $\pi$ of the risky assets is also risky in the sense that $\sigma^{2}(\pi)>0$. The solution to equation (39) is

$$
P(t)=P \exp \left\{\left(\mu(\pi)-\frac{1}{2} \sigma^{2}(\pi)\right) t+\sigma(\pi) B(t)\right\},
$$

with expectation and standard deviation given by

$$
\begin{aligned}
& E[P(t)]=P e^{\mu(\pi) t} \\
& \sigma[P(t)]=P e^{\mu(\pi) t} \sqrt{e^{\sigma^{2}(\pi) t}-1} .
\end{aligned}
$$

The stochastic differential equation (39) was derived by Merton (1971, 1990), see also Rubinstein (1991). It can also be derived using elementary arguments by taking limits of lognormal sums, see Milevsky \& Posner (1998).

Let $k$ be a strictly positive integer. Investing according to investment strategy $\pi$, an amount of 1 at time $k-1$ will grow to the random amount $e^{Y_{k}(\pi)}$ at time $k$, where $Y_{k}(\pi)$ denotes the yearly return in year $k$ of investment strategy $\pi$. One finds that

$$
Y_{k}(\pi)=\left(\mu(\pi)-\frac{1}{2} \sigma^{2}(\pi)\right)+\sigma(\pi)(B(k)-B(k-1)) .
$$


Hence, the random yearly returns $Y_{k}(\pi)$ of the constantly rebalanced portfolio $\pi$ are independent and identically distributed normal random variables with

$$
\begin{aligned}
E\left[Y_{k}(\pi)\right] & =\mu(\pi)-\frac{1}{2} \sigma^{2}(\pi), \\
\operatorname{Var}\left[Y_{k}(\pi)\right] & =\sigma^{2}(\pi) .
\end{aligned}
$$

Note that this observation about the yearly returns also holds in case $\pi$ equals the zero vector. The price $P(k)$ can be written in terms of the yearly returns as follows:

$$
P(k)=P \exp \left(Y_{1}(\pi)+Y_{2}(\pi)+\cdots+Y_{k}(\pi)\right) .
$$

\subsection{Markowitz mean-variance analysis}

In 1990, Harry M. Markowitz received the Nobel Prize in Economics (shared with William F. Sharpe and Merton H. Miller) for his theory on portfolio selection under uncertainty. The contribution for which he received the award was first published in Markowitz (1952) and more extensively in Markowitz (1959). As mentioned in the press release of the Royal Swedish Academy of Sciences, Markowitz's theory can be considered as the first approach to solving the problem that each investor faces, namely how to find the optimal trade-off between risk and return, i.e. how to find the optimal investment strategy under the two conflicting objectives of high expected return versus low risk of the investment portfolio. Markowitz proposed a way to reduce the complicated and multidimensional problem of finding the optimal portfolio with respect to a large number of different assets to a conceptual simple two-dimensional problem, known as mean-variance analysis. The Markowitz approach has become very popular due to the fact that it combines algebraic simplicity and suitability for practical applications. The mean-variance approach provides a fundamental basis for portfolio selection in a single period. A selected overview of the tremendous amount of research initiated by Markowitz's seminal work can be found in Steinbach (2001).

Several variants of the classical single-period mean-variance problem exist. Here, we will consider the formulation that we will need later on in the paper. Among all constant mix portfolios $\pi$ with a given portfolio drift $\mu(\pi)=\mu$, we look for the one with the smallest volatility $\sigma(\pi)$. Hence, for any given value of $\mu$, we want to find the solution of the following problem:

$$
\operatorname{Min}_{\pi} \sigma^{2}(\pi) \text { subject to } \mu(\pi)=\mu .
$$

We will denote the portfolio that corresponds to the minimum in (46) by $\pi^{\mu}$.

The assumption that $\mu \neq r 1$, together with the assumptions that the variance-covariance matrix is positive definite and that short-selling is allowed implies that there exists a unique local global minimum for problem (46). A Lagrange optimization yields:

$$
\sigma^{2}\left(\pi^{\mu}\right)=\frac{(\mu-r)^{2}}{(\mu-r \mathbf{1})^{T} \cdot \boldsymbol{\Sigma}^{-1} \cdot(\mu-r \mathbf{1})}
$$

and

$$
\pi^{\mu}=(\mu-r) \frac{\boldsymbol{\Sigma}^{-1} \cdot(\mu-r \mathbf{1})}{(\mu-r \mathbf{1})^{T} \cdot \boldsymbol{\Sigma}^{-1} \cdot(\mu-r \mathbf{1})}
$$


Note that $\sigma^{2}\left(\pi^{\mu}\right)$ and $\pi^{\mu}$ are well-defined, because the inverse of a positive definite matrix is also positive definite.

The efficient frontier refers to the set of all solutions $\left\{\left(\sigma\left(\pi^{\mu}\right), \mu\right)\right\}$ for the optimization problem (46). From (47) we see that the efficient frontier consists of two straight lines in the $(\sigma, \mu)$-plane:

$$
\begin{array}{ll}
\mu=r+\sqrt{(\mu-r \mathbf{1})^{T} \cdot \boldsymbol{\Sigma}^{-1} \cdot(\mu-r \mathbf{1})} \sigma\left(\pi^{\mu}\right), & \mu \geq r, \\
\mu=r-\sqrt{(\mu-r \mathbf{1})^{T} \cdot \boldsymbol{\Sigma}^{-1} \cdot(\mu-r \mathbf{1})} \sigma\left(\pi^{\mu}\right), & \mu \leq r .
\end{array}
$$

The portfolios $\pi^{\mu}$ belonging to the efficient frontier are called mean-variance efficient portfolios. Portfolios on the lower branch are irrelevant from a mean-variance optimization viewpoint as they lead to a positive volatility while their drift is lower than $r$. The upper branch $\left\{\left(\sigma\left(\pi^{\mu}\right), \mu\right) \mid \mu \geq r\right\}$ is referred to as the 'Capital Market Line'.

In the following, we will call portfolios $\pi$ that fulfill the condition $\mathbf{1}^{T} \times \pi=1$ risky-assetsonly portfolios because such portfolios consist only of risky assets. It can be proven that if we only consider risky-assets-only portfolios, the efficient frontier is a hyperbola in the mean - standard deviation space (provided there are at least two risky assets with different drift). Now consider the risky-assets-only global minimal variance portfolio $\pi^{(m)}$, i.e. the portfolio that is the solution of the following problem:

$$
\operatorname{Min}_{\pi} \sigma^{2}(\pi) \text { subject to } \mathbf{1}^{T} \cdot \pi=1 .
$$

This portfolio and its drift are given by

$$
\begin{aligned}
\pi^{(m)} & =\frac{\boldsymbol{\Sigma}^{-1} \cdot \mathbf{1}}{\mathbf{1}^{T} \cdot \boldsymbol{\Sigma}^{-1} \cdot \mathbf{1}}, \\
\mu\left(\pi^{(m)}\right) & =\frac{\mathbf{1}^{T} \cdot \boldsymbol{\Sigma}^{-1} \cdot \mu}{\mathbf{1}^{T} \cdot \boldsymbol{\Sigma}^{-1} \cdot \mathbf{1}} .
\end{aligned}
$$

One can prove that under the condition

$$
\mu\left(\pi^{(m)}\right)>r
$$

the Capital Market Line is at a tangent to the upper branch of the hyperbola that corresponds to the efficient frontier of risky-asset-only portfolios. When $\mu\left(\pi^{(m)}\right)<r$, the decreasing part of the efficient frontier (49) will be tangent to the lower branch of the hyperbola.

Let us now assume that $\mu\left(\pi^{(m)}\right) \neq r$. The portfolio that corresponds to the point of intersection between the efficient frontier (49) and the risky-assets-only efficient frontier is called the 'tangency portfolio', and is denoted by $\pi^{(t)}$. The assumption that $\mu\left(\pi^{(m)}\right) \neq r$ implies that $\mu\left(\pi^{(t)}\right) \neq r$. One can easily verify that $\pi^{(t)}$ is given by

$$
\pi^{(t)}=\frac{\boldsymbol{\Sigma}^{-1} \cdot(\mu-r \mathbf{1})}{\mathbf{1}^{T} \cdot \boldsymbol{\Sigma}^{-1} \cdot(\mu-r \mathbf{1})} .
$$

Note that (48) can be rewritten as

$$
\pi^{\mu}=\left(\frac{\mu-r}{\mu\left(\pi^{\mathbf{t}}\right)-r}\right) \pi^{(t)} .
$$


This means that every mean-variance efficient portfolio $\pi^{\mu}$ consists of a fraction $\left(\frac{\mu-r}{\mu\left(\pi^{(\mathbf{t})}\right)-r}\right)$ invested in the risky-assets-only portfolio $\pi^{(t)}$ and a fraction $\left(1-\frac{\mu-r}{\mu\left(\pi^{(t)}\right)-r}\right)$ invested in the riskfree asset. Mean-variance optimizing investors only differ in terms of which fraction of their wealth they put in the tangency portfolio.

The result that all mean-variance investors will hold only two kinds of portfolios (or mutual funds), the exclusively risky portfolio $\pi^{(t)}$ and the riskfree asset, is often called a Mutual Fund Theorem or a Two Fund Separation Theorem. Note that in case $\mu\left(\pi^{(m)}\right)=r$, there is no tangency portfolio, but any portfolio on the efficient frontier can still be constructed as a linear combination of two basic portfolios on the efficient frontier.

In case $\mu\left(\pi^{(m)}\right)>r$ is fulfilled, also the inequality $\mu\left(\pi^{(t)}\right)>r$ holds. The Capital Market Line can then be rewritten as

$$
\mu=r+\left(\frac{\mu\left(\pi^{(\mathbf{t})}\right)-r}{\sigma\left(\pi^{(t)}\right)}\right) \sigma\left(\pi^{\mu}\right)
$$

This equation describes the drift of the return for an investor as related to the volatility that he is willing to accept. The slope $\frac{\mu\left(\pi^{(\mathbf{t})}\right)-r}{\sigma\left(\pi^{t}\right)}$ is referred to as the 'Sharpe ratio'. It can be interpreted as the price of risk reduction: It shows by how much the drift increases if the volatility increases by 1 unit.

Many papers have been published that consider variants of the classical mean-variance portfolio selection criterion, where the variance is replaced by an alternative asymmetric risk measure that measures downside risk, in order to avoid penalization due to over-performance, see e.g. Emmer, Klüppelberg \& Korn (2001) or Li, Ng, Tan \& Yang (2003). Note that Markowitz (1959) already introduced the idea to replace the variance by an alternative asymmetric risk measure in a more general mean - risk approach.

The single period mean-variance model was soon extended to multiperiod portfolio selection. Research on multiperiod portfolio selection has been dominated by the idea of maximizing expected utility functions of terminal wealth. Markowitz (1959) already considered longterm investment planning by considering multiperiod models based on attaching a utility to the levels of consumption of wealth over time.

\section{Saving and terminal wealth}

\subsection{General problem description}

In this section, we will consider the problem of how to invest periodic saving amounts in order to reach some target capital at a predetermined future time $n$. Let $\alpha_{i}$ be the positive amount that will be invested at time $i,(i=0,1,2, \cdots, n)$. We assume that these amounts are invested according to a constant mix portfolio $\pi$ as defined in Section 3.2. The choice of the constant portfolio mix has to be made at time 0 . An amount of 1 unit invested at time $i$ will grow to the random amount $e^{\sum_{j=i+1}^{n} Y_{j}(\pi)}$ at time $n$. 
Let $W_{j}(\pi)$ be the wealth at time $j$, defined by the following recursive relation:

$$
W_{j}(\pi)=W_{j-1}(\pi) e^{Y_{j}(\pi)}+\alpha_{j}, \quad j=1, \cdots, n,
$$

with initial value $W_{0}(\pi)=\alpha_{0}$. Hence, $W_{j}(\pi)$ is the wealth that will be available at time $j$, including the savings amount $\alpha_{j}$ at time $j$. The realization of $W_{j}(\pi)$ will be known at time $j$, and depends on the investment returns (stochastic part) and on the savings (deterministic part) in the past. Note that the random variables $Y_{j}(\pi)$ are i.i.d. and normal distributed with parameters $\mu(\pi)$ and $\sigma(\pi)$ as defined in (40).

From the recursion (56) for the wealth process, we find the following explicit expression for terminal wealth $W_{n}(\pi)$ :

$$
W_{n}(\pi)=\sum_{i=0}^{n} \alpha_{i} e^{\sum_{j=i+1}^{n} Y_{j}(\pi)}
$$

By convention, $\sum_{i=m}^{n} b_{i}$ is set equal to 0 if $m>n$.

Within the expected utility theory framework of Von Neumann \& Morgenstern (1947), the investor could choose the investment strategy $\pi$ that maximizes his expected utility of final wealth:

$$
\max _{\pi} E\left[u\left(W_{n}(\pi)\right)\right],
$$

where $u$ is the utility function he uses to appreciate the different levels of final wealth.

Another approach, within the framework of Yaari's (1987) dual theory of choice under risk, is to choose the optimal investment strategy as the one that maximizes the distorted expectation of final wealth:

$$
\max _{\pi} \rho_{f}\left[W_{n}(\pi)\right],
$$

where $f$ is the investor's distortion function and $\rho_{f}$ is the 'distorted expectation', determined with $f\left(\operatorname{Pr}\left(W_{n}(\pi)>x\right)\right)$ :

$$
\rho_{f}\left[W_{n}(\pi)\right]=-\left(\int_{-\infty}^{0} 1-f\left(\operatorname{Pr}\left(W_{n}(\pi)>x\right)\right)\right) \mathrm{d} x+\int_{0}^{\infty} f\left(\operatorname{Pr}\left(W_{n}(\pi)>x\right)\right) \mathrm{d} x .
$$

While in utility theory, choosing among risks is performed by comparing expected values of transformed wealth levels (utilities), in Yaari's theory the quantities that are compared are the 'distorted expectations' of wealth levels. The distorted expectation of final wealth $W_{n}(\pi)$ can be interpreted as an expectation of $W_{n}(\pi)$ evaluated with a distorted probability measure' in the sense of a Choquet-integral, see Denneberg (1994). The decision maker acts in order to maximize the distorted expectation of final wealth.

For a distortion function $f_{p}, 0<p<1$, given by

$$
f_{p}(x)= \begin{cases}0 & : 0 \leq x<p \\ 1 & : p \leq x \leq 1\end{cases}
$$

we find

$$
\begin{aligned}
\rho_{f_{p}}\left[W_{n}(\pi)\right] & =Q_{1-p}^{+}\left[W_{n}(\pi)\right] \\
& =\sup \left\{x \in \mathbb{R} \mid \operatorname{Pr}\left(W_{n}(\pi)>x\right) \geq p\right\} .
\end{aligned}
$$


The optimization problem (59) with distortion function given by (61) determines the optimal investment strategy as the one that maximizes the largest amount that will be reached with a probability of at least $p$.

For the convex distortion function $g_{p}, 0<p<1$, given by

$$
g_{p}(x)=\left\{\begin{array}{cl}
0 & : 0 \leq x<p \\
\frac{x-p}{1-p} & : p \leq x \leq 1
\end{array}\right.
$$

we find

$$
\rho_{g_{p}}\left[W_{n}(\pi)\right]=C L T E_{1-p}\left[W_{n}(\pi)\right] .
$$

In Yaari's theory, a decision maker is called risk-averse if he has a convex distortion function. Hence, the optimization problem (59) with distortion function (63) can be interpreted as the problem to be solved by a risk-averse decision maker with distortion function $g_{p}$. The optimal investment strategy is the one that maximizes the conditional expected value of final wealth, given that the $p$-target capital is not reached.

For a more detailed comparison between the two theories of choice under risk and their relation to risk measures, see e.g. Dhaene, Vanduffel, Tang, Goovaerts, Kaas \& Vyncke (2004).

\subsection{The case of a single investment}

\subsubsection{Time and portfolio diversification}

Emmer, Klüppelberg \& Korn (2001) remark that there seems to be common wisdom that long term stock investment leads to an almost sure gain over locally riskfree bond investments. In the long run stock indices are growing faster than riskfree rates, despite the repeated occurrence of stock market declines. The conventional perception therefore holds that the longer the investment horizon, the greater should be one's proportion invested in risky assets. In order to verify if this common wisdom holds true, we will consider the terminal wealth problem with a single investment of 1 at time 0 . Hence, $\alpha_{0}=1$ and $\alpha_{1}=\alpha_{2}=\ldots=\alpha_{n}=0$. We will assume that one can invest in one riskfree asset $P^{0}(t)$ and in $m$ risky assets $P^{i}(t)$ as explained in section 3.1. We will also assume that $\mu\left(\pi^{(m)}\right)>r$ holds, which implies that $\mu\left(\pi^{(t)}\right)>r$.

The distribution function of final wealth $W_{n}(\pi)$ follows from

$$
W_{n}(\pi) \stackrel{d}{=} e^{n\left[\mu(\pi)-\frac{1}{2} \sigma^{2}(\pi)\right]+\sqrt{n} \sigma(\pi) \Phi^{-1}(U)} .
$$

Within the framework of expected utility theory, one determines the optimal constantly rebalanced portfolio as the one that maximizes the investor's expected utility of final wealth:

$$
\max _{\pi} E\left[u\left(W_{n}(\pi)\right)\right]
$$

For the logarithmic utility function $u(x)=\ln (x)$, one finds that the optimal portfolio $\pi^{*}$ lies on the Capital Market Line and is given by

$$
\pi^{*}=\left(\frac{\mu\left(\pi^{(t)}\right)-r}{\sigma^{2}\left(\pi^{(t)}\right)}\right) \pi^{t} .
$$


Note that within the logarithmic utility framework, the optimal strategy (67) is independent of the investment horizon. For more details, see e.g. Merton (1990).

Within Yaari's dual theory of choice under risk, let us now consider the optimization problem

$$
\max _{\pi} Q_{1-p}^{+}\left[W_{n}(\pi)\right]
$$

with $\frac{1}{2}<p<1$. The quantiles of final wealth $W_{n}(\pi)$ are given by

$$
Q_{1-p}^{+}\left[W_{n}(\pi)\right]=Q_{1-p}\left[W_{n}(\pi)\right]=e^{n\left(\mu(\pi)-\frac{1}{2} \sigma^{2}(\pi)\right)-\sqrt{n} \sigma(\pi) \Phi^{-1}(p) .}
$$

One can easily verify that for a fixed value of $\mu(\pi)$, the quantile is decreasing in $\sigma(\pi)$. On the other hand, for a fixed value of $\sigma(\pi)$, the quantile is increasing in $\mu(\pi)$, implying that the optimal portfolio of (68) will correspond to a point on the Capital Market Line and is given by

$$
\pi^{*}=\left(\frac{\mu\left(\pi^{t}\right)-r}{\sigma^{2}\left(\pi^{t}\right)}-\frac{\Phi^{-1}(p)}{\sqrt{n} \sigma\left(\pi^{t}\right)}\right)_{+} \pi^{t}, \quad \frac{1}{2}<p<1,
$$

where $(x)_{+}$stands for $\max (0, x)$. From $(70)$ it follows that in this setting, increasing the investment time horizon transforms the optimal investment strategy into a more risk-taking one. Hence, investors with a longer time horizon should have a larger exposure to stocks relative to investors with a shorter time horizon. The optimal risky proportion converges to the optimal growth portfolio (67). We can conclude that in case the time horizon becomes infinitely large, the optimal growth portfolio will outperform any other portfolio, with respect to optimality criterion (68).

Next, consider the optimization problem of a risk averse decision maker who determines the optimal investment strategy as the solution of the following maximization problem:

$$
\max _{\pi} C L T E_{1-p}\left[W_{n}(\pi)\right]
$$

From (16) we find that

$$
C L T E_{1-p}\left(W_{n}(\pi)\right)=e^{n \mu(\pi)} \frac{1-\Phi\left(\sqrt{n} \sigma(\pi)+\Phi^{-1}(p)\right)}{1-p}
$$

One can again verify that for a fixed value of $\mu(\pi)$, the $C L T E_{1-p}$ is decreasing in $\sigma(\pi)$, while for a fixed value of $\sigma(\pi)$, it is increasing in $\mu(\pi)$. This implies that the optimum of problem (71) will also correspond to a point on Capital Market Line.

Another way to look at the time diversification effect is to consider the 'Equity Shortfall Risk' of the investment portfolio. Following Milevsky (2003), we define the Equity Shortfall Risk over a given investment period $n$ and for a given investment strategy $\pi$ by

$$
\operatorname{ESR}(\pi, n)=\operatorname{Pr}\left(W_{n}(\pi) \leq e^{n r}\right) .
$$

Hence, $\operatorname{ESR}(\pi, n)$ is the probability that the risky investment strategy $\pi$ will underperform the riskfree investment strategy over a time horizon $n$. It can be interpreted as the probability of regretting the investment, where the investor regrets his choice $\pi$ compared to the riskfree strategy if this last strategy will have performed better than the risky investment strategy. 
The concept of Expected Shortfall Risk was introduced in the financial literature by Roy (1952), in a one-period discrete-time setting.

A straightforward calculation leads to

$$
\operatorname{ESR}(\pi, n)=1-\Phi\left(\sqrt{n}\left(\frac{\mu(\pi)-r}{\sigma(\pi)}-\frac{1}{2} \sigma(\pi)\right)\right) .
$$

From this expression, we see that, provided $\mu(\pi)-\frac{1}{2} \sigma(\pi)^{2}>r$, increasing the time horizon $n$ will decrease $\operatorname{ESR}(\pi, n)$. Moreover, when the time horizon goes to infinity, the Expected Shortfall Risk disappears and the risky investment strategy $\pi$ will outperform the riskfree investment strategy with probability 1 . Important to note however is that when the portfolio variance $\sigma(\pi)^{2}$ becomes large relative to $\mu(\pi)-r, \operatorname{ESR}(\pi, n)$ increases with the time horizon and reaches level 1 at infinity. We can conclude that the general perception of time-diversification expressed in terms of decreasing Expected Shortfall Risk is in accordance with the theory, provided the expected yearly returns $E\left[Y_{k}(\pi)\right]$ exceed the risk free yearly return $r$.

Comparing the optimal investment strategies (67) and (70), we can conclude that the time diversification benefit strongly depends on the optimality criterion that is considered. It has to be mentioned that the belief in time-diversification is not general and that the (non-) existence of a time-diversifying benefit is the subject of a heavy debate. The topic is considered in Samuelson (1989), Marshall (1994), Bodie (1995), Jagganathan \& Kocherlakota (1996) and Milevsky (2003), amongst others.

Milevsky (2003) also considers the concept of space diversification, by which he means the diversification effect caused by increasing the number of risky assets in the investment portfolio. Provided $\mu(\pi)>r$, we find that decreasing the portfolio volatility $\sigma(\pi)$ will, ceteris paribus, lead to an increase of the argument in the $\Phi($.$) function in (74). Hence,$ any increase of the number of risky assets which allows to reduce the portfolio volatility while keeping the portfolio drift constant (or increasing it) will decrease $\operatorname{ESR}(\pi, n)$. In other words, a better space-diversified portfolio implies a lower Equity Shortfall Risk.

This phenomenon can easily be illustrated in the case of a homogeneous market (i.e. all securities have equal drift and variance, all correlations are equal and positive) and applying the 'naive' constant mix strategy where all proportions are kept equal: $\pi_{i}=\frac{1}{m}$. In this particular case, it is straightforward to prove that increasing the number of securities $m$ will keep the portfolio drift constant while decreasing the portfolio variance.

The interrelationship and trade-off between the two possible dimensions of diversification, i.e. the number of stocks in a portfolio and the number of periods over which an investment is held, is investigated in detail in Milevsky (2003).

\subsubsection{Numerical illustration}

Consider a Black \& Scholes market with a riskfree asset with a yearly return $r=0.03$ and two risky assets with yearly drifts equal to $\mu_{1}=0.06$ and $\mu_{2}=0.10$ respectively. The volatilities of the risky assets are given by $\sigma_{1}=0.10$ and $\sigma_{2}=0.20$. Pearson's correlation 


\begin{tabular}{c|ccccc}
\hline$p$ & $n=1$ & $n=10$ & $n=20$ & $n=40$ & $n=100$ \\
\hline 0.99 & 0 & 0 & 0 & 0.33 & 1.23 \\
0.97 & 0 & 0 & 0 & 0.80 & 1.52 \\
0.95 & 0 & 0 & 0.33 & 1.04 & 1.68 \\
0.90 & 0 & 0.08 & 0.87 & 1.43 & 1.92 \\
\hline
\end{tabular}

Table 1: Optimal portfolios $\pi^{*}$ in case of maximizing $Q_{1-p}\left[W_{n}(\pi)\right]$

\begin{tabular}{c|ccccc}
\hline$p$ & $n=1$ & $n=10$ & $n=20$ & $n=40$ & $n=100$ \\
\hline 0.99 & 0 & 0 & 0 & 0 & 0.96 \\
0.97 & 0 & 0 & 0 & 0.43 & 1.37 \\
0.95 & 0 & 0 & 0 & 0.47 & 1.50 \\
0.90 & 0 & 0 & 0 & 0 & 0.96 \\
\hline
\end{tabular}

Table 2: Optimal portfolios $\pi^{*}$ in case of maximizing $C L T E_{1-p}\left[W_{n}(\pi)\right]$

coefficient $\frac{\sigma_{12}}{\sigma_{1} \sigma_{2}}$ is given by 0.5 . From (53) we find that the tangency portfolio is given by $\pi^{(t)}=\left(\frac{5}{9}, \frac{4}{9}\right)$ with drift $\mu\left(\pi^{(t)}\right)=7 / 90$ and volatility $\sigma\left(\pi^{(t)}\right)=\sqrt{\frac{43}{2700}}$.

We consider a single investment at time 0 . In Table 1 and Table 2 we present the optimal risky proportions $\pi^{*}$ with respect to the optimization problems (68) and (71), for different values of the probability level $p$ and the investment period $n$.

The figures in Table 1 and Table 2 illustrate that increasing the time horizon leads to an increased optimal proportion invested in the risky asset. Also, the lower the probability level $p$ with which we want to reach the target, the higher the proportion to be invested in the risky asset. Finally observe that the maximization of $C L T E_{1-p}\left[W_{n}(\pi)\right]$ leads to lower optimal risky proportions as compared to the maximization of $Q_{1-p}^{+}\left[W_{n}(\pi)\right]$.

\subsection{Comonotonic approximations for the general problem}

Let us now consider the general terminal wealth problem as described in Section 4.1. From (57), we see that $W_{n}(\pi)$ is a sum of non-independent lognormal random variables. As it is impossible to determine the distribution function of $W_{n}(\pi)$ analytically, we will derive a convex order upper bound $W_{n}^{c}(\pi)$ and a convex order lower bound $W_{n}^{l}(\pi)$ for $W_{n}(\pi)$.

Rewriting $W_{n}(\pi)$ as

$$
W_{n}(\pi)=\sum_{i=0}^{n} \alpha_{i} e^{Z_{i}}
$$

we see that we can apply the results of Section 2.3 with

$$
\begin{aligned}
Z_{i} & =Y_{i+1}(\pi)+Y_{i+2}(\pi)+\cdots+Y_{n}(\pi), \\
E\left[Z_{i}\right] & =(n-i)\left[\mu(\pi)-\frac{1}{2} \sigma^{2}(\pi)\right], \\
\sigma_{Z_{i}}^{2} & =(n-i) \sigma^{2}(\pi) .
\end{aligned}
$$


It follows that the comonotonic upper bound $W_{n}^{c}(\pi)$ for $W_{n}(\pi)$ is given by

$$
W_{n}^{c}(\pi)=\sum_{i=0}^{n} \alpha_{i} e^{(n-i)\left[\mu(\pi)-\frac{1}{2} \sigma^{2}(\pi)\right]+\sqrt{n-i} \sigma(\pi) \Phi^{-1}(U)} .
$$

For $p \in(0,1)$, the quantiles of $W_{n}^{c}(\pi)$ are given by

$$
Q_{1-p}^{+}\left[W_{n}^{c}(\pi)\right]=Q_{1-p}\left[W_{n}^{c}(\pi)\right]=\sum_{i=0}^{n} \alpha_{i} e^{(n-i)\left[\mu(\pi)-\frac{1}{2} \sigma^{2}(\pi)\right]-\sqrt{n-i} \sigma(\pi) \Phi^{-1}(p)}
$$

while $C L T E_{p}\left[W_{n}^{c}(\pi)\right]$ is given by

$$
C L T E_{1-p}\left[W_{n}^{c}(\pi)\right]=\sum_{i=0}^{n} \alpha_{i} e^{(n-i) \mu(\pi)} \frac{1-\Phi\left(\sqrt{n-i} \sigma(\pi)+\Phi^{-1}(p)\right)}{1-p} .
$$

In order to define a convex lower bound $W_{n}^{l}(\pi)$ for $W_{n}(\pi)$, we choose the conditioning random variable as follows:

$$
\Lambda(\pi)=\sum_{j=1}^{n} \beta_{j}(\pi) Y_{j}(\pi)
$$

where the coefficients $\beta_{j}(\pi)$ follow from (24). Notice that the lower bound approximation

$$
W_{n}^{l}(\pi)=E\left[W_{n}(\pi) \mid \Lambda(\pi)\right]
$$

is only determined up to a linear transformation of $\Lambda(\pi)$. Hence, we propose the following coefficients:

$$
\beta_{j}(\pi)=\sum_{k=0}^{j-1} \alpha_{k} e^{-k \mu(\pi)}
$$

for the conditioning random variable $\Lambda(\pi)$. It follows that for this choice of the parameters $\beta_{j}(\pi)$, the variance of the lower bound will be close to the variance of $W_{n}(\pi)$, provided $\sigma^{2}(\pi)$ is small enough.

From Section 2.3, we find

$$
W_{n}^{l}(\pi)=\sum_{i=0}^{n} \alpha_{i} e^{(n-i) \mu(\pi)-\frac{1}{2} r_{i}^{2}(\pi)(n-i) \sigma^{2}(\pi)+r_{i}(\pi) \sqrt{n-i} \sigma(\pi) \Phi^{-1}(U)}
$$

where the coefficients $r_{i}(\pi)$ are given by

$$
r_{i}(\pi)=\frac{\sum_{j=i+1}^{n} \sum_{k=0}^{j-1} \alpha_{k} e^{-k \mu(\pi)}}{\sqrt{n-i} \sqrt{\sum_{j=1}^{n}\left(\sum_{k=0}^{j-1} \alpha_{k} e^{-k \mu(\pi)}\right)^{2}}} .
$$

Note that the correlation coefficients $r_{i}(\pi)$ are non-negative which implies that $W^{l}(\pi)$ is a comonotonic sum of lognormal random variables.

The following expression can be derived for the risk measure $Q_{1-p}^{+}\left(W_{n}^{l}(\pi)\right), p \in(0,1)$ :

$$
Q_{1-p}^{+}\left[W_{n}^{l}(\pi)\right]=Q_{1-p}\left[W_{n}^{l}(\pi)\right]=\sum_{i=0}^{n} \alpha_{i} e^{(n-i)\left(\mu(\pi)-\frac{1}{2} r_{i}^{2}(\pi) \sigma^{2}(\pi)\right)-r_{i}(\pi) \sqrt{n-i} \sigma(\pi) \Phi^{-1}(p)}
$$


while for $C L T E_{1-p}\left[W_{n}^{l}(\pi)\right]$ we find

$$
C L T E_{1-p}\left[W_{n}^{l}(\pi)\right]=\sum_{i=0}^{n} \alpha_{i} e^{(n-i) \mu(\pi)} \frac{1-\Phi\left(r_{i}(\pi) \sqrt{n-i} \sigma(\pi)+\Phi^{-1}(p)\right)}{1-p} .
$$

From Theorem 2.1 we find that

$$
-W_{n}^{l}(\pi) \leq_{c x}-W_{n}(\pi) \leq_{c x}-W_{n}^{c}(\pi) .
$$

This implies that

$$
C T E_{p}\left[-W_{n}^{l}(\pi)\right] \leq C T E_{p}\left[-W_{n}(\pi)\right] \leq C T E_{p}\left[-W_{n}^{c}(\pi)\right], \quad 0<p<1,
$$

see e.g. Dhaene, Vanduffel, Goovaerts, Kaas, Vyncke (2004). Using (6) we find

$$
C L T E_{1-p}\left[W_{n}^{c}(\pi)\right] \leq C L T E_{1-p}\left[W_{n}(\pi)\right] \leq C L T E_{1-p}^{l}\left[W_{n}^{c}(\pi)\right], \quad 0<p<1 .
$$

Note however that the approximations $Q_{1-p}\left(W_{n}^{l}(\pi)\right)$ and $Q_{1-p}\left(W_{n}^{c}(\pi)\right.$ for the quantiles $Q_{1-p}\left(W_{n}(\pi)\right)$ are not necessarily ordered in the same way.

\subsection{Determining the investment strategy that maximizes the tar- get capital, for a given probability level}

\subsubsection{The $p$ - target capital}

For a given probability level $\frac{1}{2}<p<1$ and a given investment strategy $\pi$, we define the $p$-target capital $K$ as the $(1-p)$-th order " + "-quantile of terminal wealth:

$$
K=Q_{1-p}^{+}\left[W_{n}(\pi)\right] \text {. }
$$

One immediately finds that

$$
K=\sup \left\{x \in \mathbb{R} \mid \operatorname{Pr}\left[W_{n}(\pi)>x\right] \geq p\right\} .
$$

Hence, the target capital at probability level $p$ can be interpreted as the maximal amount that will be available at time $n$, with a probability of at least $p$.

Now assume that a probability level $p$ is fixed and that the optimal investment strategy $\pi^{*}$ is determined as the one that maximizes the $p$ - target capital. Denoting the optimal target capital by $K^{*}$, we have

$$
K^{*}=\max _{\pi} Q_{1-p}^{+}\left[W_{n}(\pi)\right] .
$$

Note that from (59) and (62), it follows that this optimization problem can be interpreted in terms of Yaari's dual theory of choice under risk.

Solving (92) is from a computational point of view a complicated problem because of the multi-dimensionality involved. Indeed, a 'time-dimensionality' occurs because $W_{n}(\pi)$ is a sum of $n$ dependent accumulation factors. There is also a 'portfolio-dimensionality' involved as the maximum has to be determined over all portfolios $\pi$. In the following sections we will show how to get rid of this 'curse of dimensionality'. 


\subsubsection{The comonotonic upper bound for $W_{n}(\pi)$}

As it is impossible to determine $Q_{1-p}^{+}\left(W_{n}(\pi)\right)$ analytically, we propose to approximate $\pi^{*}$ by $\pi^{c}$, where $\pi^{c}$ is the investment strategy that maximizes $Q_{1-p}^{+}\left(W_{n}^{c}(\pi)\right)$. The optimal target capital $K^{*}$ is approximated by $K^{c}$, which is given by:

$$
K^{c}=\max _{\pi} Q_{1-p}^{+}\left[W_{n}^{c}(\pi)\right] .
$$

As we assumed that $\frac{1}{2}<p<1$, it follows from (78) that for a given value of $\mu(\pi)$, the quantile $Q_{p}\left(W_{n}^{c}(\pi)\right)$ is a decreasing function of $\sigma(\pi)$. Hence, the (approximate) optimal portfolio $\pi^{c}$ can be found on the efficient frontier (49). On the other hand, for a given value of $\sigma(\pi)$, the quantile $Q_{p}\left(W_{n}^{c}(\pi)\right)$ is an increasing function of $\mu(\pi)$. This implies that the portfolio $\pi^{c}$ will correspond to a point on the Capital Market Line. The solution of the general maximization problem is then found to be the portfolio on the Capital Market Line that maximizes the quantile $Q_{1-p}^{+}\left(W_{n}^{c}(\pi)\right.$ :

$$
K^{c}=\max _{\mu \geq r} Q_{1-p}^{+}\left[W_{n}^{c}\left(\pi^{\mu}\right)\right],
$$

where the portfolio $\pi^{\mu}$ is given by (48).

\subsubsection{The comonotonic lower bound for $W_{n}(\pi)$}

We also propose to approximate the optimal investment strategy $\pi^{*}$ by $\pi^{l}$, where $\pi^{l}$ is the investment strategy that maximizes $Q_{1-p}^{+}\left(W_{n}^{l}(\pi)\right)$. The $p$ - target capital $K^{*}$ is then approximated by $K^{l}$, which is given by

$$
K^{l}=\max _{\pi} Q_{1-p}^{+}\left[W_{n}^{l}(\pi)\right] .
$$

It follows from (85) that for a given value of $\mu(\pi)$, the correlation coefficient is fixed and the quantile $Q_{p}\left(W_{n}^{l}(\pi)\right)$ is a decreasing function of $\sigma(\pi)$. Hence, $\pi^{l}$ is an element of the set of efficient portfolios. The general maximization problem can be reduced to the following maximization problem:

$$
K^{l}=\max _{\mu} Q_{1-p}^{+}\left[W_{n}^{l}\left(\pi^{\mu}\right)\right] .
$$

The approximated optimization problems (94) and (96) solve the curse of dimensionality. The multi-dimensionality caused by time $n$ is reduced to one dimension by introducing the comonotonic dependency structure. Also the portfolio-dimensionality $m$ is reduced to one dimension because the optimal solutions are to be found on the efficient frontier.

\subsubsection{Constant savings amounts}

In this subsection, we consider the special case that the saving amounts are constant. For each investment strategy $\pi$ we look for the required periodic saving amount $\alpha$ that leads to a $p$-target capital equal to 1 . From (90) we find that this saving amount $\alpha$ is given by

$$
\alpha(\pi)=\frac{1}{Q_{1-p}^{+}\left[\bar{W}_{n}(\pi)\right]},
$$


with $\bar{W}_{n}(\pi)$ given by

$$
\bar{W}_{n}(\pi)=\sum_{i=0}^{n} e^{Y_{i+1}(\pi)+Y_{i+2}(\pi)+\cdots+Y_{n}(\pi)} .
$$

The optimal investment strategy is now defined as the one that minimizes the period savings. Denoting the minimal saving amount by $\alpha^{*}$, we have

$$
\alpha^{*}=\min _{\pi} \alpha(\pi)
$$

Note that in the case of constant saving amounts, the investment strategy that maximizes the $p$ - target capital $K$ for given saving amounts $\alpha$ is identical to the investment strategy that minimizes the periodic savings $\alpha$ for a given target capital $K$.

Now we approximate $\bar{W}_{n}(\pi)$ by $\bar{W}_{n}^{c}(\pi)$ as explained in (77). The minimal periodic savings amount $\alpha^{*}$ is then approximated by $\alpha^{c}$ which is given by

$$
\alpha^{c}=\min _{\mu} \frac{1}{Q_{1-p}^{+}\left[\bar{W}_{n}^{c}\left(\pi^{\mu}\right)\right]} .
$$

Next, we approximate $\bar{W}_{n}(\pi)$ by $\bar{W}_{n}^{l}(\pi)$ as explained in (83). The minimal periodic savings amount $\alpha^{*}$ is then approximated by $\alpha^{l}$ which is given by

$$
\alpha^{l}=\min _{\mu} \frac{1}{Q_{1-p}^{+}\left[\bar{W}_{n}^{l}\left(\pi^{\mu}\right)\right]} .
$$

\subsubsection{Numerical illustration}

Consider the Black \& Scholes type market with one riskfree and two risky assets as explained in Subsection 4.2.2. We assume saving amounts $\alpha_{i}=1$ for $i=0,1, \ldots, 39$, while $\alpha_{40}=0$. As we have seen, the solutions of the problems (93) and (95) are to be found on the efficient frontier. Because of the Two Fund Theorem, any portfolio on the efficient frontier can be expressed as a linear combination of the riskfree portfolio and the tangency portfolio $\pi^{(t)}$. Hence, we can reduce the market to a market consisting of one riskfree asset with $r=0.03$ and one risky asset with drift and volatility equal to the corresponding values of the tangency portfolio in the original setting.

In Figure 1 we show the comonotonic lower bound approximations $Q_{0.05}\left[W_{40}^{l}\left(\pi^{\mu}\right)\right]$ (solid line) and the comonotonic upper bound approximations $Q_{0.05}\left[W_{40}^{c}\left(\pi^{\mu}\right)\right]$ (dashed line) for the 0.95 target capital $Q_{0.05}\left[W_{40}\left(\pi^{\mu}\right)\right]$, for different values of the risky proportion $\pi^{\mu}$ invested in the tangency portfolio $\pi^{(t)}$, i.e. for the different portfolios on the mean-variance efficient set. We compare these quantiles with the simulated quantiles $Q_{0.05}\left[W_{40}^{s}\left(\pi^{\mu}\right)\right]$ (dotted line). The simulation was performed by generating 20,000 paths using antithetic variables. We observe that the lower bound approximation is very close to the results obtained by simulation. Indeed, the maximum of the relative deviations $\left|\frac{Q_{0.05}\left[W_{40}^{l}\left(\pi^{\mu}\right)\right]-Q_{0.05}\left[W_{40}^{s}\left(\pi^{\mu}\right)\right]}{Q_{0.05}\left[W_{40}^{s}\left(\pi^{\mu}\right)\right]}\right|$ is less than $0.9 \%$. The maximum of the respective curves correspond to the (approximated or simulated) optimal portfolio.

For the comonotonic lower bound approximation we find that the optimal 0.95 - target capital $K^{l}$ is given by $K^{l}=89.78$. This target capital corresponds to a fraction $\pi^{l}=0.92$ 


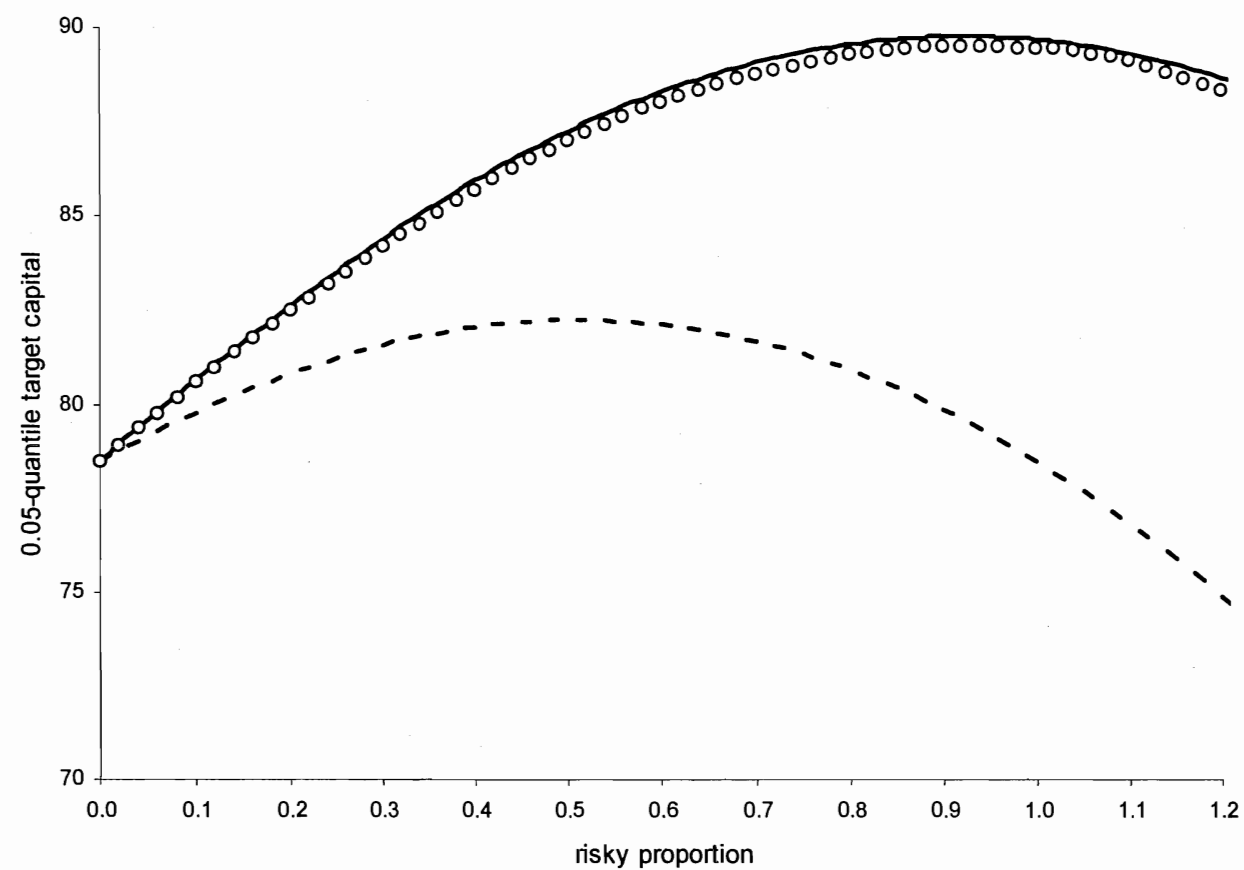

Figure 1: The approximated target capitals $Q_{0.05}\left[W_{40}^{l}\left(\pi^{\mu}\right)\right]$ (solid line), $Q_{0.05}\left[W_{40}^{c}\left(\pi^{\mu}\right)\right]$ (dashed line) and the simulated target capital $Q_{0.05}\left[W_{40}^{s}\left(\pi^{\mu}\right)\right]$ (dotted line) as a function of the risky proportion $\pi^{\mu}$. 


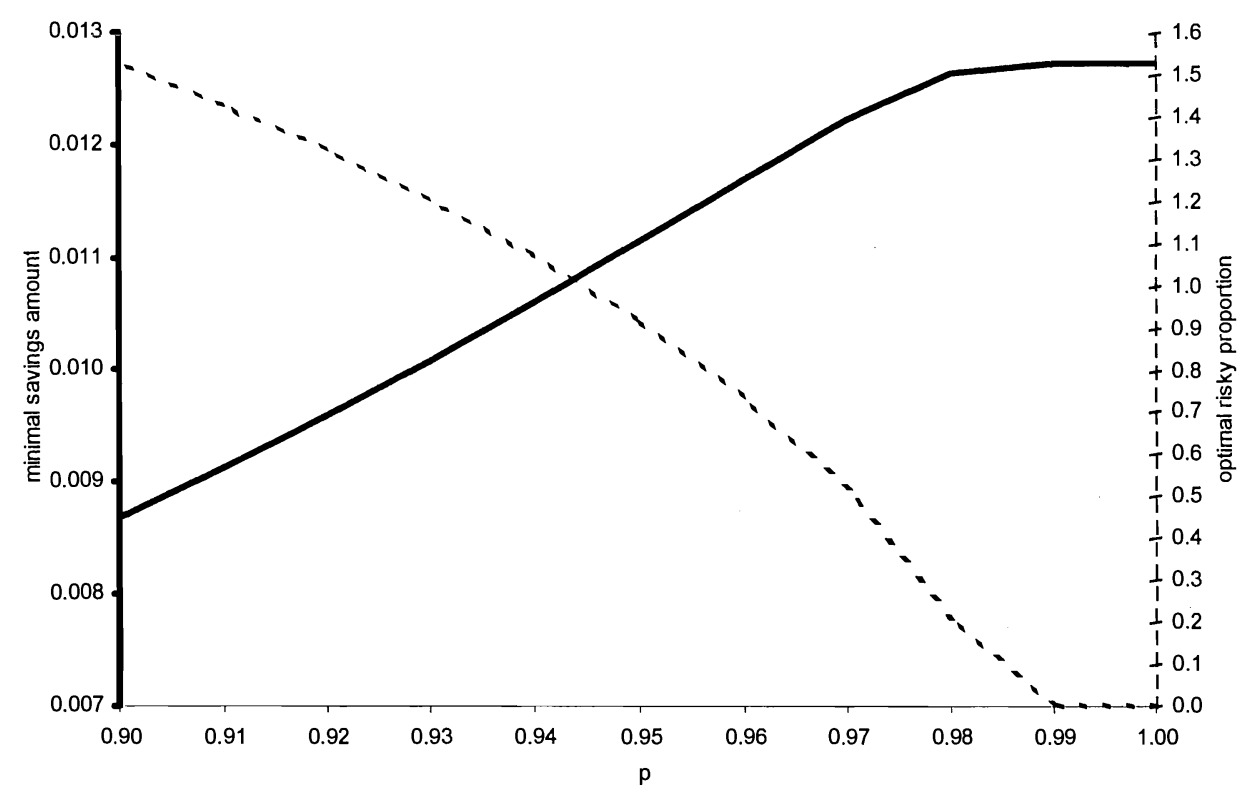

Figure 2: The minimal savings amount $\alpha^{l}$ (solid line - left scale) and the optimal risky proportion $\pi^{l}$ (dashed line - right scale) as a function of $p$.

invested in the tangency portfolio.

The comonotonic upper bound approximation gives rise to an optimum $\left(\pi^{c}, K^{c}\right)=(0.51,82.25)$. The simulated optimum is reached in $\left(\pi^{s}, K^{s}\right)=(0.92,89.52)$.

We can conclude that the lower bound approximation for the optimal investment strategy performs very well, compared to the simulated solution. From Figure 1 we also see that increasing the risky proportion increases the target capital until a certain level. Further increasing the investment in the risky asset decreases the target capital again. This observation is in accordance with intuition about optimal investment strategies.

In Figure 2, we consider the same market as above. We assume constant saving amounts $\alpha$ at times $0,1, \ldots, 39$ and a target capital equal to 1 to be reached at time 40 . We consider the investment strategy that minimizes the yearly savings amount for different probability levels $p$ of the target capital. The computations were performed with the lower bound approximation $\bar{W}_{40}^{l}(\pi)$ for $\bar{W}_{40}(\pi)$.

The solid line represents the (approximated) minimal savings amount $\alpha^{l}$ for different probability levels $p$ of a target capital equal to 1 (left scale). As we see from the figure, increasing the required probability of reaching the target of 1 , increases the optimal savings amount. Note that the required savings amount in case of the riskfree investment, i.e. the one that corresponds to $p=1$, is given by 0.0127 .

The dashed line represents the (approximated) optimal risky proportion $\pi^{l}$ to be invested in the tangency portfolio, for different probability levels $p$ (right scale). As could be expected, increasing the probability of reaching the target capital decreases the optimal risky proportion in the portfolio. 


\subsubsection{Maximizing the CLTE}

The optimal investment strategy $\pi^{*}$ can also be defined as the one that maximizes the CLTE for a given value of $p$ :

$$
C \operatorname{CLT} E_{1-p}\left[W_{n}\left(\pi^{*}\right)\right]=\max _{\pi} C L T E_{1-p}\left[W_{n}(\pi)\right] .
$$

Note that this optimization problem can be interpreted in terms of Yaari's dual theory of choice under risk. It is the problem faced by a risk averse decision maker with distortion function (64) who wants to optimize the distorted expectation of his final wealth. The optimization problem in this case can be expressed as follows: the decision maker with target capital $Q_{1-p}^{+}\left(W_{n}(\pi)\right)$ maximizes the expected value of final wealth, given that the target capital is not reached.

Approximating $W_{n}(\pi)$ by $W_{n}^{l}(\pi)$ or $W_{n}^{c}(\pi)$ and using the results of the previous sections, leads to approximate solutions similar to the one derived above for problem (92). Indeed, the CLTE's of both approximations can be written as sums of CLTE's of lognormal random variables. The $n$-dimensional maximization problem can again be reduced to a one-dimensional optimization problem over all portfolios on the mean-variance efficient set. Derivation of the results is left as an exercise to the reader.

\subsection{Determining the investment strategy that maximizes the prob- ability level for a given target capital.}

\subsubsection{The probability of reaching the target}

In this subsection, we will assume that the target capital $K>0$ is given. For a given investment strategy $\pi$, the probability of reaching this target is given by

$$
p=\bar{F}_{W_{n}(\pi)}(K),
$$

where $\bar{F}_{W_{n}(\pi)}(x)=1-F_{W_{n}(\pi)}(x)=\operatorname{Pr}\left(W_{n}(\pi)>x\right)$. Now we propose to determine the optimal investment strategy $\pi^{*}$ as the one that maximizes the probability of reaching the target $K$. Denoting this optimal probability level by $p^{*}$, we have that

$$
p^{*}=\max _{\pi} \bar{F}_{W_{n}(\pi)}(K) \text {. }
$$

One possible choice for the target capital $K$ is the final wealth that would arise if all savings were invested in the riskfree asset:

$$
K^{r}=\sum_{i=0}^{n} \alpha_{i} e^{(n-i) r}
$$

Extending definition (73), the equity shortfall risk of a given investment strategy is now defined as

$$
\operatorname{ESR}(\pi, n)=F_{W_{n}(\pi)}\left(K^{r}\right),
$$

which is the probability that the investment strategy will underperform the riskfree investment strategy. Solving the maximization problem (104) with $K=K^{r}$ comes down to finding the investment strategy that minimizes the equity shortfall risk. 


\subsubsection{The comonotonic upper bound for $W_{n}(\pi)$}

Neither the decumulative distribution function $\bar{F}_{W_{n}(\pi)}(x)$, nor its quantiles can be determined analytically. Therefore, we will introduce the comonotonic approximations for $\bar{F}_{W_{n}(\pi)}(K)$.

The approximation $\pi^{c}$ for the the optimal investment strategy $\pi^{*}$ of the problem (104) is defined as the investment strategy that maximizes $\bar{F}_{W_{n}^{c}(\pi)}(K)$. The approximation $p^{c}$ for the optimal probability level $p^{*}$ is then given by

$$
p^{c}=\max _{\pi} \bar{F}_{W_{n}^{c}(\pi)}(K) .
$$

For any investment strategy $\pi$, with $\sigma(\pi)>0$, it follows from $(78)$ that $Q_{p}\left(W_{n}^{c}(\pi)\right)$ is a continuous and strictly increasing function of $p$, mapping $(0,1)$ in $(0, \infty)$. This implies that $F_{W_{n}^{c}(\pi)}(x)$ is a strictly increasing and continuous function of $x$. Hence, for any $K$, we find that $F_{W_{n}^{c}(\pi)}(K)$ is the unique solution of the equation $Q_{F_{W_{n}^{c}(\pi)(K)}}=K$, which can be written as

$$
\sum_{i=0}^{n} \alpha_{i} e^{(n-i)\left[\mu(\pi)-\frac{1}{2} \sigma^{2}(\pi)\right]-\sqrt{n-i} \sigma(\pi) \Phi^{-1}\left(\bar{F}_{W_{n}^{c}(\pi)}(K)\right)}=K .
$$

Now we assume that the target capital $K$ is small enough, in the sense that there exists at least one portfolio $\pi$ such that $\bar{F}_{W_{n}^{c}(\pi)}(K) \geq \frac{1}{2}$. Then it can be proven from (108) that the solution of the maximization problem (107) can be found among the portfolios on the efficient frontier:

$$
p^{c}=\max _{\mu} \bar{F}_{W_{n}^{c}\left(\pi^{\mu}\right)}(K)
$$

\subsubsection{The comonotonic lower bound for $W_{n}(\pi)$}

On the other hand, the approximation $\pi^{l}$ for $\pi^{*}$ is the investment strategy that maximizes $\bar{F}_{W_{n}^{l}(\pi)}(K)$. The approximation $p^{l}$ for the optimal probability level $p^{*}$ is then given by

$$
p^{l}=\max _{\pi} \bar{F}_{W_{n}^{l}(\pi)}(K) .
$$

For any investment strategy $\pi$, with $\sigma(\pi)>0$, it follows from $(85)$ that $Q_{p}\left(W_{n}^{l}(\pi)\right)$ is a continuous and strictly increasing function of $p$, mapping $(0,1)$ in $(0, \infty)$. This implies that $F_{W_{n}^{l}(\pi)}(x)$ is a strictly increasing and continuous function of $x$. Hence, for any $K$, we find that $F_{W_{n}^{l}(\pi)}(K)$ is the unique solution of the equation $Q_{F_{W_{n}^{l}(\pi)(K)}}=K$, which is equivalent to

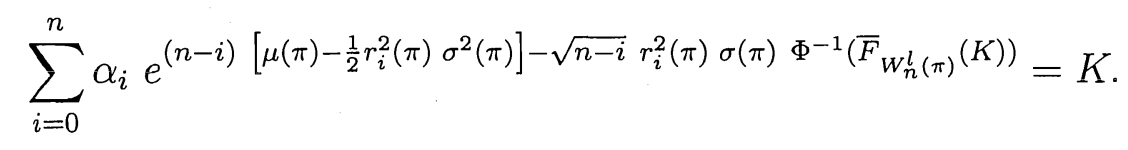

Under the assumption that $K$ is small enough, in the sense that there exists at least one portfolio $\pi$ such that $\bar{F}_{W_{n}^{l}(\pi)}(K)>\frac{1}{2}$, it can be proven that th maximization problem (110) reduces to the following one-dimensional optimization problem:

$$
p^{l}=\max _{\mu} \bar{F}_{W_{n}^{l}\left(\pi^{\mu}\right)}(K) .
$$

We can conclude that the (time- and portfolio-) curse of dimensionality of optimal investment problem (104) can also be reduced by the comonotonic approximations. 


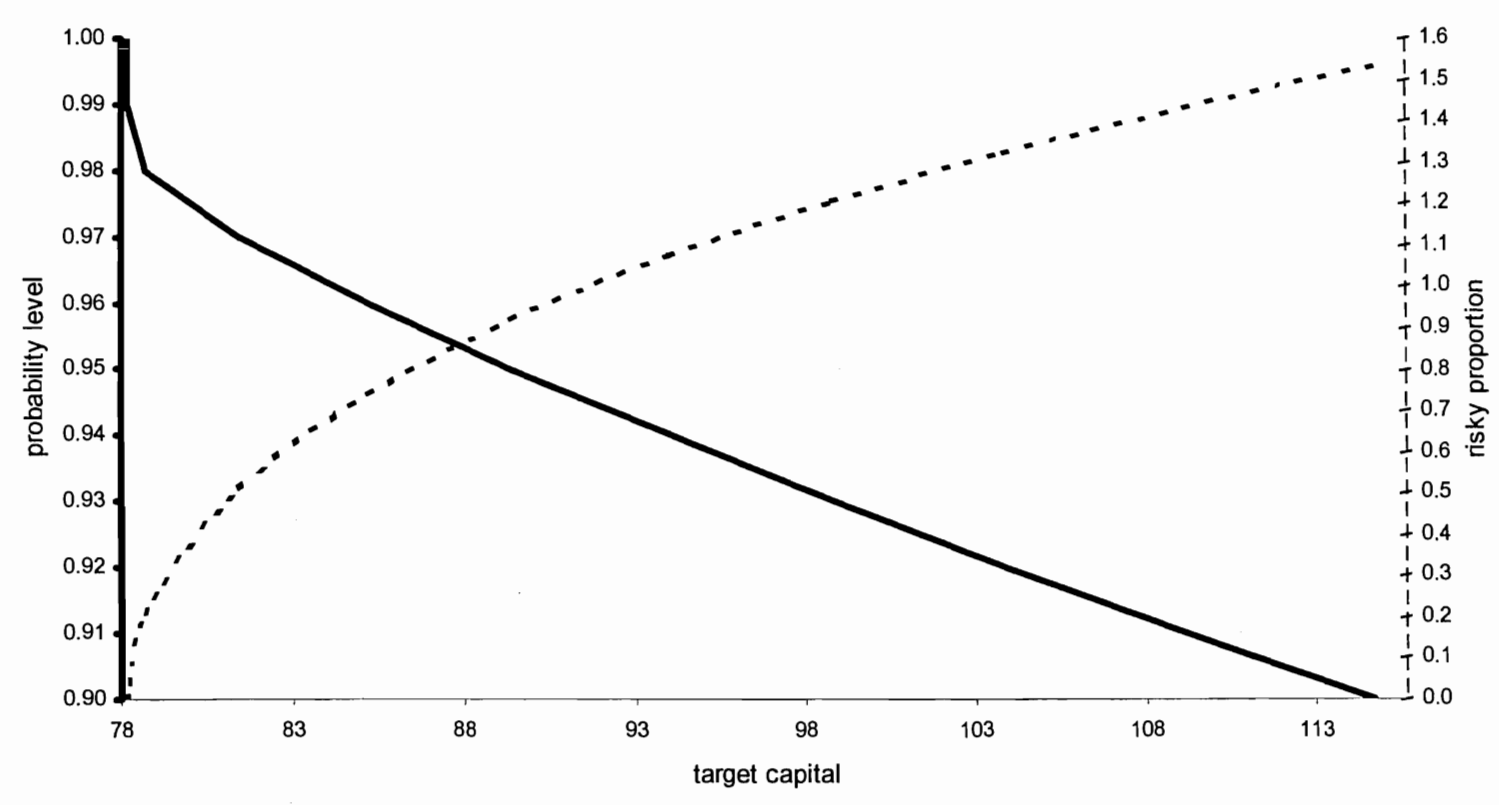

Figure 3: The maximal probability $p^{l}$ of reaching the target capital (solid line - left scale) and the optimal risky proportion $\pi^{l}$ (dashed line - right scale) as a function of the target capital $K$.

\subsubsection{Numerical illustration}

Assume the Black \& Scholes market as described in Subsection 4.2.2. Consider saving amounts $\alpha_{i}=1$ for $i=0,1, \ldots, 39$, while $\alpha_{40}=0$. We want to determine the solution of problem (110) for different values of the target capital $K$.

Provided $K$ is small enough, the solution of $(110)$ is to be found on the efficient frontier. Hence, it suffices to consider portfolios being linear combinations of the riskfree asset and the tangency portfolio $\pi^{(t)}$.

In Figure 3 we show the maximal probability $p^{l}$ of reaching the target capital and the optimal risky proportion $\pi^{l}$ to be invested in the tangency portfolio, as a function of the target capital $K$. The solid line represents the (approximated) maximal probability levels $p^{l}$ of reaching a given target capital $K$ (left scale), whereas the dashed line represents the (approximated) corresponding optimal risky proportion $\pi^{l}$ (right scale). The figure shows that increasing the level of the target capital leads to decreasing optimal probability levels and increasing risky proportions. Note that the riskfree investment corresponds to a target capital equal to 78.50 that is reached with probability equal to 1 . 


\section{$5 \quad$ Reserves for future obligations}

\subsection{General problem description}

Consider a given set of deterministic obligations, i.e. a series of deterministic non-negative payments $\alpha_{1}, \alpha_{2}, \cdots, \alpha_{n}$, that are due at times $1,2, \cdots, n$, respectively. Being able to meet these future obligations requires an appropriate funding, this means that appropriate assets has to be available to set up a reserve and a solvency margin. Following Atkinson \& Dallas (2000), the reserve is defined as the amount of funds that have to be set aside as a liability in order to meet future obligations, whereas the solvency margin is the capital that regulators, rating agencies or the company itself deem necessary for the company to be able to withstand reasonable fluctuations in financial results.

At current time 0 , assets of value $R_{0}$ are set up in order to be able to meet these future obligations. We will call $R_{0}$ the initial reserve. It can be interpreted as the reserve as defined above, or also as the total balance sheet requirement, i.e. reserve and solvency margin. We will assume that this reserve can be invested according to one of the constant mix portfolios $\pi$ as defined in the Section 3.2. This investment strategy has to be chosen at time 0 . Starting from the initial reserve $R_{0}$ and investing according to $\pi$, we define $R_{j}\left(R_{0}, \pi\right)$ at time $j$ by the following recursion:

$$
R_{j}\left(R_{0}, \pi\right)=R_{j-1}\left(R_{0}, \pi\right) e^{Y_{j}(\pi)}-\alpha_{j}, \quad j=1, \cdots, n
$$

with $R_{0}\left(R_{0}, \pi\right)=R_{0}$. Hence, $R_{j}\left(R_{0}, \pi\right)$ is the value of the assets that will be available at time $j$, after the payment of $\alpha_{j}$, given that $R_{0}\left(R_{0}, \pi\right)=R_{0}$ is the initial reserve at time 0 . The realization of $R_{j}\left(R_{0}, \pi\right)$ will be known at time $j$, and depends on the investment returns (stochastic part) and on the payments (deterministic part) in the past years. Often we will call $R_{j}\left(R_{0}, \pi\right)$ the (retrospective) reserve available at time $j$. Note that the random variables $Y_{j}(\pi)$ are i.i.d. and normal distributed with parameters $\mu(\pi)$ and $\sigma(\pi)$ as defined in (40). Solving the recursion (113), we find that the value of the assets available at time $n$ is given by

$$
R_{n}\left(R_{0}, \pi\right)=R_{0} e^{\sum_{j=1}^{n} Y_{j}(\pi)}-\sum_{i=1}^{n} \alpha_{i} e^{\sum_{j=i+1}^{n} Y_{j}(\pi)} .
$$

The random variable $S_{j}(\underline{\pi})$ is defined as the stochastically discounted value of all future payment obligations from time $j$ on, given that the investment strategy is $\underline{\pi}$ :

$$
S_{j}(\pi)=\sum_{i=j+1}^{n} \alpha_{i} e^{-\left(Y_{j+1}(\pi)+Y_{2}(\pi)+\cdots+Y_{i}(\pi)\right)} .
$$

This random variable will be called the 'stochastic future obligations' at time $j$. The following relation holds between $S_{j}(\pi), R_{j}\left(R_{0}, \pi\right)$ and $R_{n}\left(R_{0}, \pi\right)$ :

$$
R_{n}\left(R_{0}, \pi\right)=\left(R_{j}\left(R_{0}, \pi\right)-S_{j}(\pi)\right) e^{Y_{j+1}(\pi)+\cdots+Y_{n}(\pi)} .
$$

This relation implies that

$$
R_{n}\left(R_{0}, \pi\right) \geq 0 \Leftrightarrow R_{j}\left(R_{0}, \pi\right) \geq S_{j}(\pi), \quad j=0, \cdots, n-1 .
$$


Hence,

$$
\begin{aligned}
\operatorname{Pr}\left[R_{n}\left(R_{0}, \pi\right) \geq 0\right] & =\operatorname{Pr}\left[R_{j}\left(R_{0}, \pi\right) \geq S_{j}(\pi), j=0, \cdots, n-1\right] \\
& =F_{S(\pi)}\left(R_{0}\right) .
\end{aligned}
$$

Results similar to (118), applied in an obligation-based solvency framework for pension annuities can be found in Olivieri \& Pitacco (2003). Results in case of 'deterministic future obligations' can be found in Vanduffel, Dhaene, Goovaerts \& Kaas (2003).

An investor will be interested in the probability $p=\operatorname{Pr}\left[R_{n}\left(R_{0}, \pi\right) \geq 0\right]$ of "reaching the finish", for different choices of the reserve $R_{0}$ and the investment strategy $\pi$.

For a given initial reserve $R_{0}$, one could look for the investment strategy $\underline{\pi}$ that maximizes the probability level $p$. Or, for a given probability level $p$, one could look for the investment strategy $\underline{\pi}$ that minimizes the initial reserve $R_{0}$. These problems will be considered in the following sections.

\subsection{The case of a single obligation}

In this subsection, we consider the special case of setting a reserve at time 0 for a single payment obligation at time $n$. Hence, $\alpha_{1}=\alpha_{2}=\alpha_{n-1}=0$ and $\alpha_{n}=1$. The distribution function of the stochastic provision $S_{0}(\pi)$ follows from

$$
S_{0}(\pi) \stackrel{d}{=} e^{-n}\left(\mu(\pi)-\frac{1}{2} \sigma^{2}(\pi)\right)+\sqrt{n} \sigma(\pi) \Phi^{-1}(U) .
$$

For a given investment strategy $\pi$ and a given probability level $p$ with $\frac{1}{2}<p<1$, we determine the initial reserve $R_{0}$ as the $p$-quantile of $S(\pi)$ :

$$
R_{0}=Q_{p}\left[S_{0}(\pi)\right]
$$

This reserving principle clearly makes sense, as one can easily prove that

$$
Q_{p}\left[S_{0}(\pi)\right]=\inf \left\{x \mid \operatorname{Pr}\left[R_{n}(x, \pi) \geq 0\right] \geq p\right\} .
$$

This means that for a given investment strategy $\pi$, the $p$-quantile reserving principle determines the initial reserve as the "smallest" amount such that the probability of "reaching the finish" is at least $p$. From (15) it follows that the quantile $Q_{p}\left[S_{0}(\pi)\right]$ is given by

$$
Q_{p}\left[S_{0}(\pi)\right]=e^{-n\left(\mu(\pi)-\frac{1}{2} \sigma^{2}(\pi)\right)+\sqrt{n} \sigma(\pi) \Phi^{-1}(p)} .
$$

The optimal investment strategy $\underline{\pi}^{*}$ is defined as the one that minimizes $Q_{p}\left[S_{0}(\pi)\right]$. The initial provision $R_{0}^{*}$ is then set equal to this minimal quantile:

$$
R_{0}^{*}=\min _{\pi} Q_{p}\left[S_{0}(\pi)\right]
$$

As

$$
Q_{p}\left[S_{0}(\pi)\right]=\frac{1}{Q_{1-p}^{+}\left[W_{n}(\pi)\right]}
$$

with $Q_{1-p}^{+}\left[W_{n}(\pi)\right]$ given by (69), we find that the optimal portfolio of problem (123) is identical to the optimal portfolio of problem (68). Hence, $\pi^{*}$ is given by (70).

We can conclude that increasing the investment time horizon transforms the optimal investment strategy into a more risk-taking one. Investors with a longer time horizon should have a larger exposure to stocks relative to investors with a shorter time horizon. The optimal risky proportion converges to (67), which corresponds to the so-called optimal growth portfolio. 


\subsection{Comonotonic approximations for the general case}

From (118) we see that in order to compute the "probability of reaching the finish" $\operatorname{Pr}\left[R_{n}\left(R_{0}, \pi\right) \geq 0\right]$ for a given pair $\left(R_{0}, \pi\right)$, we have to determine the d.f. of $S_{0}(\pi)$. However, the random variable $S_{0}(\pi)$ is a linear combination of dependent lognormal random variables. This implies that it is impossible to determine the distribution function of $S_{0}(\pi)$ analytically. Therefore, we will consider a convex order upper bound $S^{c}(\pi)$ and a convex order lower bound $S^{l}(\pi)$ for $S(\pi)$. Rewriting $S_{0}(\pi)$ as

$$
S_{0}(\pi)=\sum_{i=1}^{n} \alpha_{i} e^{Z_{i}}
$$

we can apply the results of Subsection 2.3 with

$$
\begin{aligned}
Z_{i} & =-Y_{1}(\pi)-Y_{2}(\pi)-\cdots-Y_{i}(\pi), \\
E\left[Z_{i}\right] & =-i\left[\mu(\pi)-\frac{1}{2} \sigma^{2}(\pi)\right], \\
\sigma_{Z_{i}}^{2} & =i \sigma^{2}(\pi) .
\end{aligned}
$$

The comonotonic upper bound $S_{0}^{c}(\pi)=\sum_{i=1}^{n} F_{\alpha_{i} e^{z_{i}}}^{-1}(U)$ for $S_{0}(\pi)$ is given by

$$
S_{0}^{c}(\pi)=\sum_{i=1}^{n} \alpha_{i} \exp \left[-i \mu(\pi)+\frac{1}{2} i \sigma^{2}(\pi)+\sqrt{i} \sigma(\pi) \Phi^{-1}(U)\right],
$$

while for $p \in(0,1)$, the quantiles and $C T E$ 's of $S_{0}^{c}(\pi)$ are given by

$$
\begin{gathered}
Q_{p}\left[S_{0}^{c}(\pi)\right]=\sum_{i=1}^{n} \alpha_{i} e^{-i \mu(\pi)+\frac{1}{2} i \sigma^{2}(\pi)+\sqrt{i} \sigma(\pi) \Phi^{-1}(p)} \\
C T E_{p}\left[S_{0}^{c}(\pi)\right]=\sum_{i=1}^{n} \alpha_{i} e^{-i \mu(\pi)+i \sigma^{2}(\pi)} \frac{\Phi\left(\sqrt{i} \sigma(\pi)-\Phi^{-1}(p)\right)}{1-p}
\end{gathered}
$$

In order to define a convex lower bound $S_{0}^{l}(\pi)$ for $S_{0}(\pi)$, we choose a conditioning random variable as follows:

$$
\Lambda(\pi)=\sum_{j=1}^{n} \beta_{j}(\pi) Y_{j}(\pi)
$$

From (24) it follows that the optimal coefficients are given by $-\sum_{k=j}^{n} \alpha_{k} e^{k\left(-\mu(\pi)+\sigma^{2}(\pi)\right)} \approx$ $-\sum_{k=j}^{n} \alpha_{k} e^{-k \mu(\pi)}$. Therefore, we propose the following coefficients:

$$
\beta_{j}(\pi) \approx-\sum_{k=j}^{n} \alpha_{k} e^{-k \mu(\pi)}
$$

It follows that for this choice of the parameters $\beta_{j}(\pi)$, the variance of the lower bound will be close to the variance of $S_{0}(\pi)$, provided $\sigma^{2}(\pi)$ is small enough. Hence, the lower bound

$$
S_{0}^{l}(\pi)=E\left[S_{0}(\pi) \mid \Lambda(\pi)\right]
$$


will have a distribution function that is close to the distribution function of $S_{0}(\pi)$, provided $\sigma(\pi)$ is small enough. From Section 2.3, we find

$$
S_{0}^{l}(\pi)=\sum_{i=1}^{n} \alpha_{i} e^{-i \mu(\pi)+\left(1-\frac{1}{2} r_{i}^{2}(\pi)\right) i \sigma^{2}(\pi)+r_{i}(\pi) \sqrt{i} \sigma(\pi) \Phi^{-1}(U)}
$$

where the coefficients $r_{i}(\pi)$ are given by

$$
r_{i}(\pi)=\frac{\sum_{j=1}^{i} \sum_{k=j}^{n} \alpha_{k} e^{-k \mu(\pi)}}{\sqrt{i} \sqrt{\sum_{j=1}^{n}\left(\sum_{k=j}^{n} \alpha_{k} e^{-k \mu(\pi)}\right)^{2}}} .
$$

Note that the correlation coefficients $r_{i}(\pi)$ are non-negative. This implies that $S^{l}(\pi)$ is a comonotonic sum of lognormal random variables. From Section 2.3, we find the following expressions for the risk measures $Q_{p}\left(S_{0}^{l}(\pi)\right)$ and $C T E_{p}\left(S_{0}^{l}(\pi)\right), p \in(0,1)$ :

$$
\begin{gathered}
Q_{p}\left[S_{0}^{l}(\pi)\right]=\sum_{i=1}^{n} \alpha_{i} e^{-i \mu(\pi)+\left(1-\frac{1}{2} r_{i}^{2}(\pi)\right) i \sigma^{2}(\pi)+r_{i}(\pi) \sqrt{i} \sigma(\pi) \Phi^{-1}(p)} \\
C T E_{p}\left[S_{0}^{l}(\pi)\right]=\sum_{i=1}^{n} \alpha_{i} e^{-i \mu(\pi)+i \sigma^{2}(\pi)} \frac{\Phi\left(r_{i}(\pi) \sqrt{i} \sigma(\pi)-\Phi^{-1}(p)\right)}{1-p}
\end{gathered}
$$

From Theorem 2.1 we find that

$$
S_{0}^{l}(\pi) \leq_{c x} S_{0}(\pi) \leq_{c x} S_{0}^{c}(\pi)
$$

This implies that

$$
C T E_{p}\left[S_{0}^{l}(\pi)\right] \leq C T E_{p}\left[S_{0}(\pi)\right] \leq C T E_{p}\left[S_{0}^{c}(\pi)\right]
$$

see e.g. Dhaene, Vanduffel, Goovaerts, Kaas \& Vyncke (2004). Note however that the approximations $Q_{p}\left[S_{0}^{l}(\pi)\right]$ and $Q_{p}\left[S_{0}^{c}(\pi)\right]$ for $Q_{p}\left[S_{0}(\pi)\right]$ are not necessarily ordered in the same way.

\subsection{Determining the investment strategy that minimizes the $p$ - quantile initial reserve for a given probability level}

\subsubsection{The $p$-quantile reserving principle}

As in Section 5.2, we set the initial reserve for a given investment strategy $\pi$ and a given probability level $p$, with $\frac{1}{2}<p<1$, equal to the $p$-quantile of $S_{0}(\pi)$ :

$$
R_{0}=Q_{p}\left[S_{0}(\pi)\right]
$$

As we noted in Section 5.2, the $p$-quantile reserving principle determines the initial reserve as the "smallest" amount such that the probability of "reaching the finish" is at least $p$, see (121). 
For a given probability level $p$, we now determine the optimal investment strategy $\pi^{*}$ as the one that minimizes $Q_{p}\left[S_{0}(\pi)\right]$. The initial reserve $R_{0}^{*}$ is then set equal to this minimal quantile:

$$
R_{0}^{*}=\min _{\pi} Q_{p}\left[S_{0}(\pi)\right]
$$

Similar to the optimization problem (92), the optimization problem (137) suffers from a 'curse of dimensionality', both in time (as $S_{0}(\pi)$ is a sum of $n$ terms) and in portfolio size (as $\pi$ is an $m$-dimensional vector). The dimensionality problem will be solved by introducing comonotonic approximations for the stochastic provision $S_{0}(\pi)$.

\subsubsection{The comonotonic upper bound for $S_{0}(\pi)$}

First, we propose to approximate the optimal investment strategy $\pi^{*}$ by $\pi^{c}$, which is the investment strategy that minimizes $Q_{p}\left[S_{0}^{c}(\pi)\right]$, and we approximate the initial provision $R_{0}^{*}$ by $R_{0}^{c}$ which follows from:

$$
R_{0}^{c}=\min _{\pi} Q_{p}\left[S_{0}^{c}(\pi)\right]
$$

where the quantiles $Q_{p}\left[S_{0}^{c}(\pi)\right]$ are given by $(128)$.

As we assumed that $\frac{1}{2}<p<1$, it follows from (128) that for a given value of $\mu(\pi)$, the quantile $Q_{p}\left[S_{0}^{c}(\pi)\right]$ is an increasing function of $\sigma(\pi)$. Hence, the portfolio $\pi^{c}$ can be found on the efficient frontier (49). Further, for a given value of $\sigma(\pi)$, the quantile is decreasing in $\mu(\pi)$ which implies that $\pi^{c}$ can be found of the Capital Market Line:

$$
\left.R_{0}^{c}=\min _{\mu \geq r} Q_{p}\left[S_{0}^{c}\left(\pi^{\mu}\right)\right]\right)
$$

\subsubsection{The comonotonic lower bound for $S_{0}(\pi)$}

Secondly, we propose the approximation $\pi^{l}$ for $\pi^{*}$, where $\pi^{l}$ is the investment strategy that minimizes $Q_{p}\left[S_{0}^{l}(\pi)\right]$, and we approximate the initial provision $R_{0}^{*}$ by $R_{0}^{l}$ which follows from

$$
R_{0}^{l}=\min _{\pi} Q_{p}\left[S_{0}^{l}(\pi)\right]
$$

The quantiles $Q_{p}\left[S_{0}^{l}(\pi)\right]$ are given by (133).

Note that the correlations $r_{i}(\pi)$ defined in (132) are non-negative and constant for a given value of $\mu(\pi)$. From (133) we find that for a given value of $\mu(\pi)$ also $Q_{p}\left[S_{0}^{l}(\pi)\right]$ is an increasing functions of $\sigma(\pi)$. Finding the approximation $\pi^{l}$ for the optimal investment strategy again boils down to finding the minimal quantile among all efficient portfolios $\pi^{\mu}$ :

$$
R_{0}^{l}=\min _{\mu} Q_{p}\left[S_{0}^{l}\left(\pi^{\mu}\right)\right]
$$

The approximations (138) and (139) reduce both the time- and portfolio multidimensionality of problem (137) to dimension 1. 


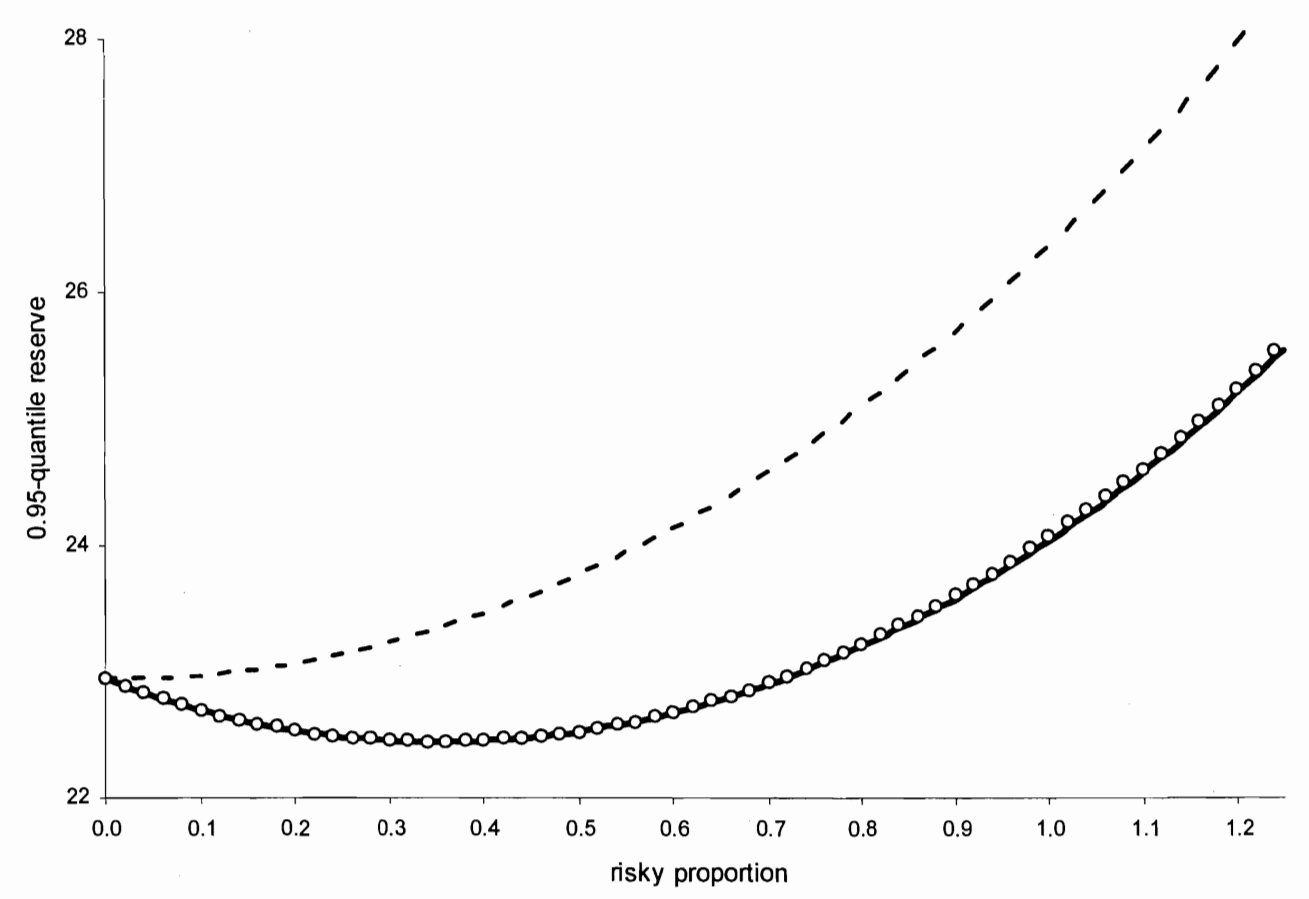

Figure 4: The approximated reserves $Q_{0.95}\left[S^{l}\left(\pi^{\mu}\right)\right]$ (solid line) and $Q_{0.95}\left[S^{c}\left(\pi^{\mu}\right)\right]$ (dashed line) and the simulated reserve $Q_{0.95}\left[S^{s}\left(\pi^{\mu}\right)\right]$ (dotted line) as a function of the risky proportion $\pi^{\mu}$.

\subsubsection{Numerical illustration}

Consider the Black \& Scholes market as considered in Section 4.2.2. consisting of one riskfree asset and two risky assets. We want to solve (approximately) problem (137) in case of a series of obligations $\alpha_{i}$ that are all equal to 1 , for $i=1, \ldots, 40$. As the solutions of the problems (138) and (139) are to be found on the efficient frontier, we can again restrict to the case of a market consisting of the riskfree asset and a risky asset that corresponds to the tangency portfolio.

In Figure 4, the comonotonic lower bound approximation $Q_{0.95}\left[S_{0}^{l}\left(\pi^{\mu}\right)\right]$ and the comonotonic upper bound approximation $Q_{0.95}\left[S_{0}^{c}\left(\pi^{\mu}\right)\right]$ are given for different values of the risky proportion $\pi^{\mu}$ invested in the tangency portfolio. Also the corresponding simulated quantiles $Q_{0.95}\left[S_{0}^{s}\left(\pi^{\mu}\right)\right]$ are given. The simulation is obtained by generating 20,000 paths using antithetic variables. We can conclude that the lower bound approximation performs extremely well. Indeed, the maximal value of the relative deviation $\left|\frac{Q_{0.95}\left[S_{0}^{l}\left(\pi^{\mu}\right)\right]-Q_{0.95}\left[S_{0}^{s}\left(\pi^{\mu}\right)\right]}{Q_{0.95}\left[S_{0}^{S}\left(\pi^{\mu}\right)\right]}\right|$ was found to be as small as $0.12 \%$.

The minimum of the respective curves corresponds to the (approximated or simulated) optimal portfolio. For the comonotonic lower bound approximation we find that the optimal proportion invested in the tangency portfolio is given by $\pi^{l}=0.35$. This corresponds to an optimal initial reserve given by $R_{0}^{l}=22.442$.

The comonotonic upper bound approximation gives rise to an optimum $\left(\pi^{c}, R_{0}^{c}\right)=(0.015,22.945)$. 


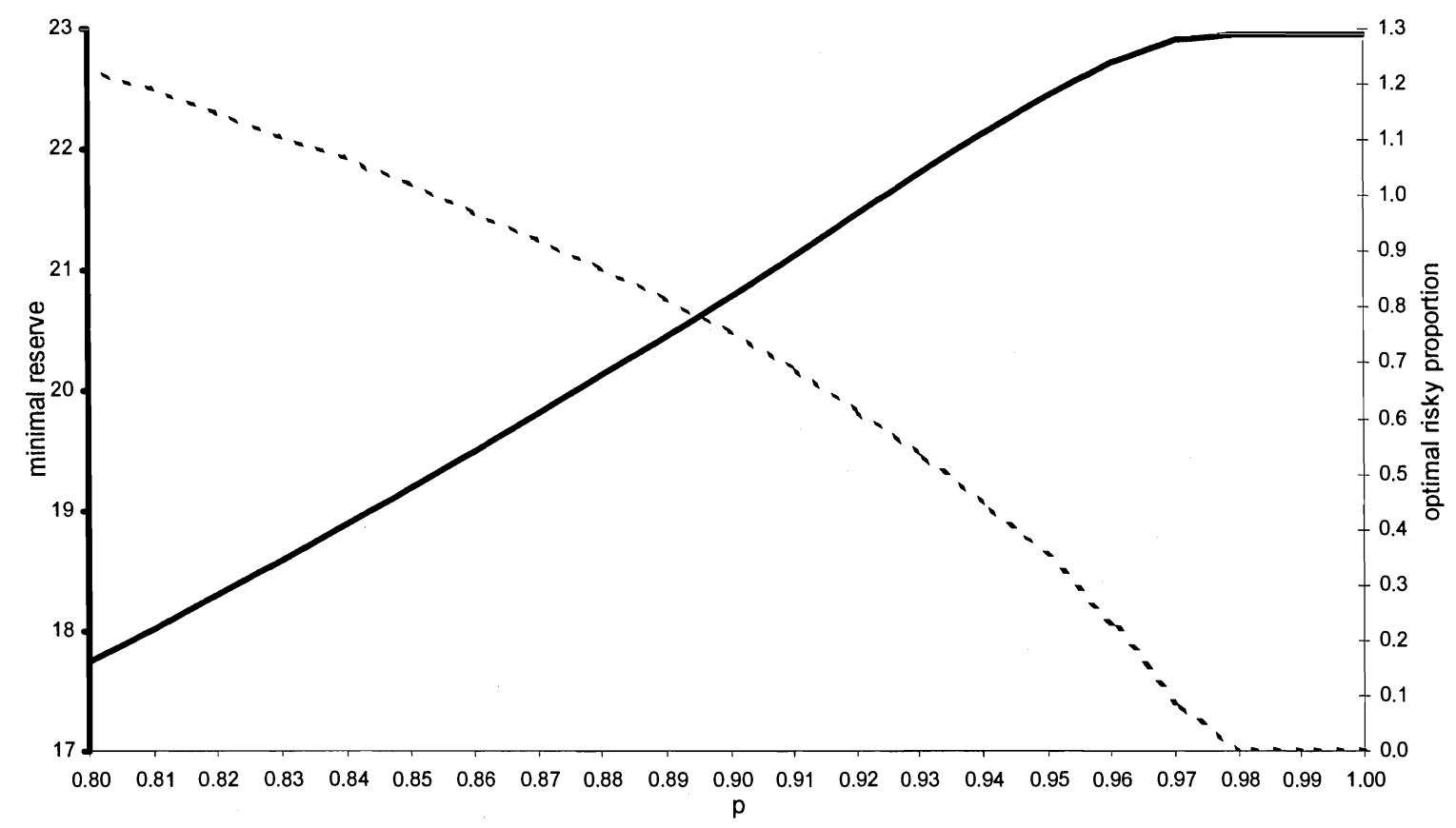

Figure 5: The minimal $Q_{p}$-reserve $R_{0}^{l}$ (solid line - left scale) and the optimal risky proportion $\pi^{l}$ (dashed line - right scale) as a function of $p$.

The simulated optimal values are given by $\left(\pi^{s}, R_{0}^{s}\right)=(0.345,22.444)$.

In Figure 5, we consider the same obligations pattern and determine the investment strategy that minimizes the initial $p$-quantile reserve for different probability levels $p$. The computations were performed using the lower bound approximation $S_{0}^{l}(\pi)$ for $S_{0}(\pi)$. The dashed line represents the (approximated) optimal risky proportion $\pi^{l}$ to be invested in the tangency portfolio, for different probability levels $p$ (right scale). The solid line represents the (approximated) initial reserve for different probability levels $p$ (left scale). In accordance with intuition, we find that increasing the probability of reaching the finish will increase the optimal initial reserve and decrease the optimal risky proportion.

Note that if one sets the probability level $p$ equal to 1 , the investment strategy is completely riskfree. The initial reserve is in this case given by 22.946 .

\subsection{Determining the investment strategy that minimizes the $C T E_{p^{-}}$ quantile initial reserve for a given probability level}

\subsubsection{The $C T E_{p}$-quantile initial reserve}

In this subsection we will set the initial reserve, for a given probability level $p \in\left(\frac{1}{2}, 1\right)$, and a given investment strategy $\pi$, equal to $C T E_{p}(S(\pi))$ :

$$
R_{0}=C T E_{p}\left[S_{0}(\pi)\right]
$$


One can prove that

$$
C T E_{p}\left[S_{0}(\pi)\right]=Q_{p}\left[S_{0}(\pi)\right]+\left|E\left[R_{n} e^{-Y_{1}(\pi)-\cdots-Y_{n}(\pi)} \mid R_{n}<0\right]\right|,
$$

where $R_{n}$ is given by

$$
R_{n}=R_{n}\left(Q_{p}\left[S_{0}(\pi)\right], \pi\right)
$$

The second term in (141) can be interpreted as the expected discounted shortfall, given that the initial reserve is set according to the $p$ - quantile reserving principle and given that there is a shortfall. Hence, with the $C T E_{p}$ reserving principle the initial reserve is set equal to the sum of the $p$ - quantile initial reserve and the expected discounted conditional shortfall of the $p$ - quantile initial reserve.

Assuming that the probability level $p$ is fixed, the optimal investment strategy $\pi^{*}$ is now determined as the one that minimizes $C T E_{p}\left[S_{0}(\pi)\right]$. The initial provision $R_{0}^{*}$ is set equal to this minimal conditional tail expectation:

$$
R_{0}^{*}=\min _{\pi} C T E_{p}\left[S_{0}(\pi)\right]
$$

As the Conditional Tail Expectations cannot be determined analytically, we again propose several approximations for this minimization problem.

\subsubsection{The comonotonic upper bound for $S_{0}(\pi)$}

We propose to approximate the optimal investment strategy $\pi^{*}$ by the investment strategy $\pi^{c}$ that minimizes $C T E_{p}\left[S_{0}^{c}(\pi)\right]$. The initial reserve $R_{0}^{*}$ is approximated by $R_{0}^{c}$ :

$$
R_{0}^{c}=\min _{\pi} C T E_{p}\left[S_{0}^{c}(\pi)\right]
$$

The quantities $C T E_{p}\left[S_{0}^{c}(\pi)\right]$ can be determined from (128).

It follows from (128) that for a given value of $\mu(\pi), C T E_{p}\left[S_{0}^{c}(\pi)\right]$ is an increasing function of $\sigma(\pi)$. Hence, finding the approximation $\pi^{c}$ for the optimal investment strategy again boils down to looking for the optimal on the mean-variance efficient frontier:

$$
R_{0}^{c}=\min _{\mu} C T E_{p}\left[S_{0}^{c}\left(\pi^{\mu}\right)\right]
$$

\subsubsection{The comonotonic lower bound for $S_{0}(\pi)$}

Next, we propose to approximate $\pi^{*}$ by $\pi^{l}$ which is the investment strategy that minimizes $C T E_{p}\left[S_{0}^{l}(\pi)\right]$. The initial reserve $R_{0}^{*}$ is then approximated by

$$
R_{0}^{l}=\min _{\pi} C T E_{p}\left[S_{0}^{l}(\pi)\right]
$$

The quantities $C T E_{p}\left[S_{0}^{l}(\pi)\right]$ are given by (133).

The $n$-dimensional minimization problem can again be reduced to the following onedimensional problem:

$$
R_{0}^{l}=\min _{\mu} C T E_{p}\left[S_{0}^{l}\left(\pi^{\mu}\right)\right] .
$$




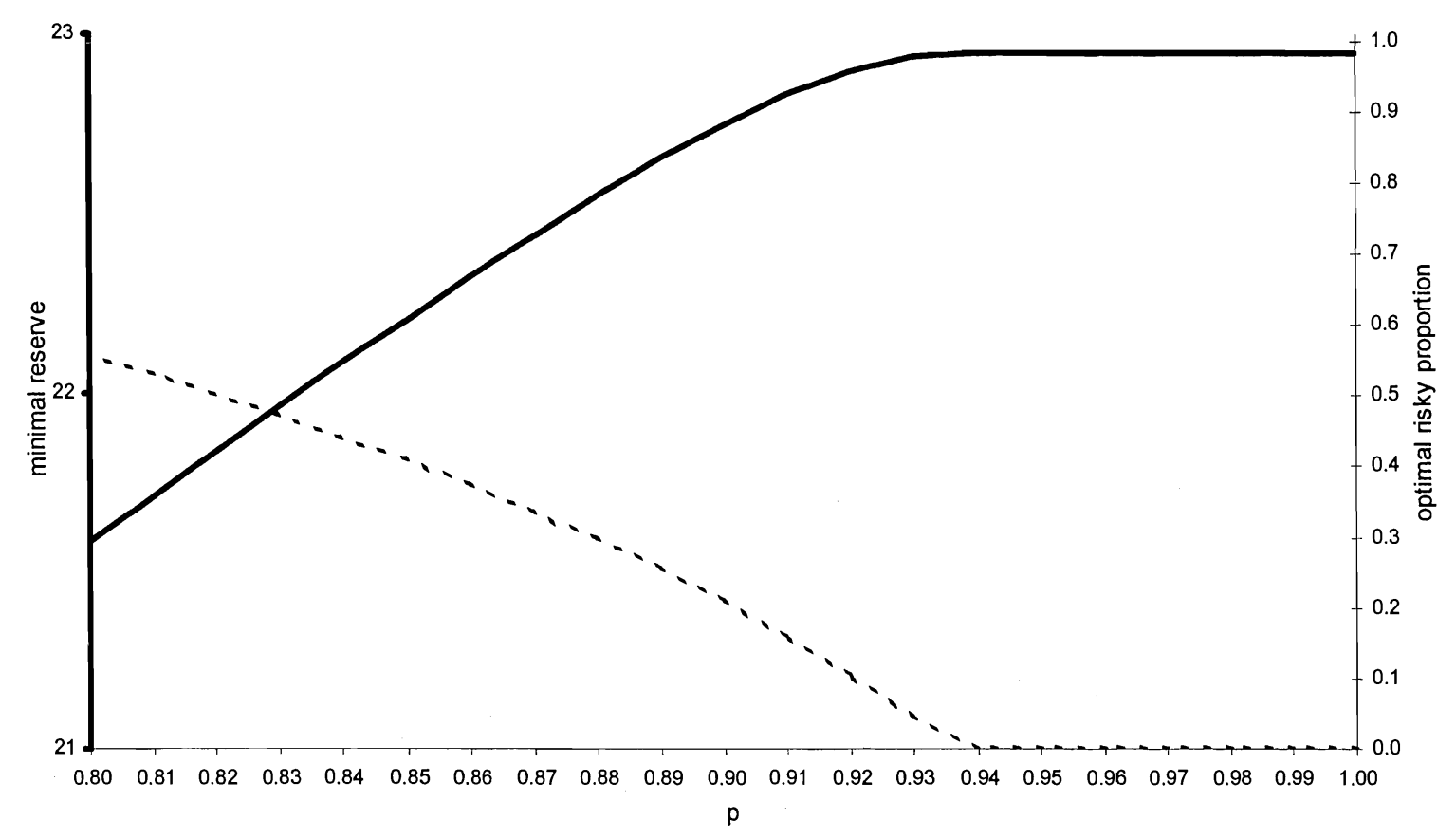

Figure 6: The minimal $C T E_{p}$-reserve $R_{0}^{l}$ (solid line - left scale) and the optimal risky proportion $\pi^{l}$ (dashed line - right scale) as a function of $p$.

It is straightforward to prove that the following inequalities hold for any investment strategy $\pi$ :

$$
C T E_{p}\left[S_{0}^{l}(\pi)\right] \leq C T E_{p}\left[S_{0}(\pi)\right] \leq C T E_{p}\left[S_{0}^{c}(\pi)\right] .
$$

These inequalities imply

$$
R_{0}^{l} \leq R_{0}^{*} \leq R_{0}^{c}
$$

\subsubsection{Numerical illustration}

Consider the Black \& Scholes market as presented in Section 4.2.2. Now we want to solve (approximately) problem (143) in case of a series of obligations $\alpha_{i}$ that are all equal to 1 , for $i=1, \ldots, 40$. As the solution of problem (147) is a portfolio on the efficient frontier, we can again restrict to the case of a market consisting of the riskfree asset and a risky asset that corresponds to the tangency portfolio.

In Figure 6, we consider the investment strategy that minimizes the initial $C T E_{p^{-}}$reserve for different values of the probability level $p$. The computations were performed with the lower bound approximation $S^{l}(\pi)$ for $S(\pi)$.

The dashed line represents the (approximated) optimal risky proportion $\pi^{l}$ to be invested in the tangency portfolio, for different probability levels $p$ (right scale). As could be expected, increasing the required probability level of reaching the finish will decrease the optimal risky proportion in the portfolio. 
The solid line represents the (approximated) $C T E$-reserve for different probability levels $p$ (left scale). As we see from the figure, increasing the probability will increase the optimal reserve.

\subsection{Determining the investment strategy that maximizes the prob- ability level, for a given initial reserve.}

\subsubsection{The probability of reaching the finish}

From (118), we find that for a given investment strategy $\pi$ and a given initial reserve $R_{0}$, the probability $p$ of "reaching the finish" is given by

$$
p=F_{S_{0}(\pi)}\left(R_{0}\right) \text {. }
$$

In this subsection, we will assume that the initial reserve $R_{0}>0$ is given. We propose to determine the optimal investment strategy $\pi^{*}$ as the one that maximizes the probability of "reaching the finish" in relation (150). Denoting this maximal probability by $p^{*}$, we find

$$
p^{*}=\max _{\pi} F_{S_{0}(\pi)}\left(R_{0}\right) .
$$

Neither the probabilities $F_{S_{0}(\pi)}\left(R_{0}\right)$, nor the quantiles $Q_{p}\left[S_{0}(\pi)\right]$ can be determined analytically. Moreover, solving optimization problem (151) by simulation is extremely timeconsuming, due to the multi-dimensionality in time and portfolio choice. Therefore, we will again propose comonotonic approximations for $\pi^{*}$ and $p^{*}$.

\subsubsection{The comonotonic upper bound for $S_{0}(\pi)$}

A first approximation consists in approximating the optimal investment strategy $\pi^{*}$ by the investment strategy $\pi^{c}$ which is the one that maximizes $F_{S_{0}^{c}(\pi)}\left(R_{0}\right)$. The probability of "reaching the finish" $p^{*}$ is then approximated by $p^{c}$, which follows from

$$
p^{c}=\max _{\pi} F_{S_{0}^{c}(\pi)}\left(R_{0}\right) .
$$

For any investment strategy $\pi$, with $\sigma(\pi)>0$, it follows from $(128)$ that $Q_{p}\left(S_{0}^{c}(\pi)\right)$ is a continuous and strictly increasing function of $p$, mapping $(0,1)$ in $(0, \infty)$. This implies that $F_{S_{0}^{c}(\pi)}(x)$ is a strictly increasing and continuous function of $x$. Hence, for any $R_{0}$, we find that $F_{S_{0}^{c}(\pi)}\left(R_{0}\right)$ is the unique solution of the equation $Q_{F_{S_{0}^{c}(\pi)}\left(R_{0}\right)}=R_{0}$, or equivalently, $F_{S_{0}^{c}(\pi)}\left(R_{0}\right)$ is the unique solution of

$$
\sum_{i=1}^{n} \alpha_{i} e^{-i \mu(\pi)+\frac{1}{2} i \sigma^{2}(\pi)+\sqrt{i} \sigma(\pi) \Phi^{-1}\left(F_{S_{0}^{c}(\pi)}\left(R_{0}\right)\right)}=R_{0} .
$$

Now, we assume that the initial provision is large enough, in the sense that there exists at least one portfolio $\pi$ such that $F_{S_{0}^{c}(\pi)}\left(R_{0}\right) \geq \frac{1}{2}$. This is equivalent to assuming that there exists at least one portfolio $\pi$ for which $E\left[Y_{k}(\pi)\right] \geq \delta$, with $\delta$ defined by

$$
\sum_{i=1}^{n} \alpha_{i} e^{-i \delta}=R_{0}
$$


Then it can be proven that the maximization problem in (152) reduces to the following maximization problem:

$$
p^{c}=\max _{\mu} F_{S_{0}^{c}\left(\pi^{\mu}\right)}\left(R_{0}\right),
$$

with $\pi^{\mu}$ the mean-variance efficient portfolio as defined in (48).

\subsubsection{The comonotonic lower bound for $S_{0}(\pi)$}

The approximation $\pi^{l}$ for $\pi^{*}$ is the investment strategy that maximizes $F_{S_{0}^{l}(\pi)}\left(R_{0}\right)$. The probability of "reaching the finish" $p^{*}$ is then approximated by $p^{l}$, which is given by

$$
p^{l}=\max _{\pi} F_{S_{0}^{l}(\pi)}\left(R_{0}\right) .
$$

Provided $\sigma(\pi)>0$, one finds that $F_{S^{l}(\pi)}\left(R_{0}\right)$ is the unique solution of the following equation:

$$
\sum_{i=1}^{n} \alpha_{i} e^{-i \mu(\pi)+\left(1-\frac{1}{2} r_{i}^{2}(\pi)\right) i \sigma^{2}(\pi)+r_{i}(\pi) \sqrt{i} \sigma(\pi) \Phi^{-1}\left(F_{S_{0}^{l}(\pi)}\left(R_{0}\right)\right)}=R_{0}
$$

Now we assume that the initial provision is large enough, in the sense that there exists at least one portfolio $\pi$ such that $F_{S_{0}^{l}(\pi)}\left(R_{0}\right) \geq \frac{1}{2}$. Then it can be proven that the maximization problem in (156) reduces to the following maximization problem:

$$
p^{l}=\max _{\mu} F_{S_{0}^{l}\left(\pi^{\mu}\right)}\left(R_{0}\right)
$$

\subsubsection{Numerical illustration}

Consider again the Black \& Scholes market as introduced in Section 4.2.2. We want to find the optimal investment strategy determined by (151) for a series of future obligations $\alpha_{i}$ that are all equal to 1 , for $i=1, \ldots, 40$. We propose to approximate the exact solution by the solution of problem (158). As the solution of problem (158) is a portfolio on the efficient frontier, we can again restrict to the case of a market consisting of the riskfree asset and a risky asset that corresponds to the tangency portfolio. In Figure 7 we show the maximal probability $p^{l}$ of reaching the finish (solid line - left scale), as well as the optimal risky proportion $\pi^{l}$ (dashed line - right scale), as a function of the initial reserve $R_{0}$.

The figure shows that increasing the level of the initial reserve will lead to an increase of the maximal probability of reaching the finish. On the other hand, increasing the initial reserve will lead to an optimal investment strategy that is less risky.

\section{Final remarks}

In this paper we considered the problem of how the available funds should be allocated among a basket of riskfree and risky assets, when the available investment strategies are to be chosen within the class of constant mix strategies. Two general asset allocation decision problems were distinguished. The terminal wealth problem considers the optimal investment mix in a situation where at regular points in time saving amounts are added to the available 


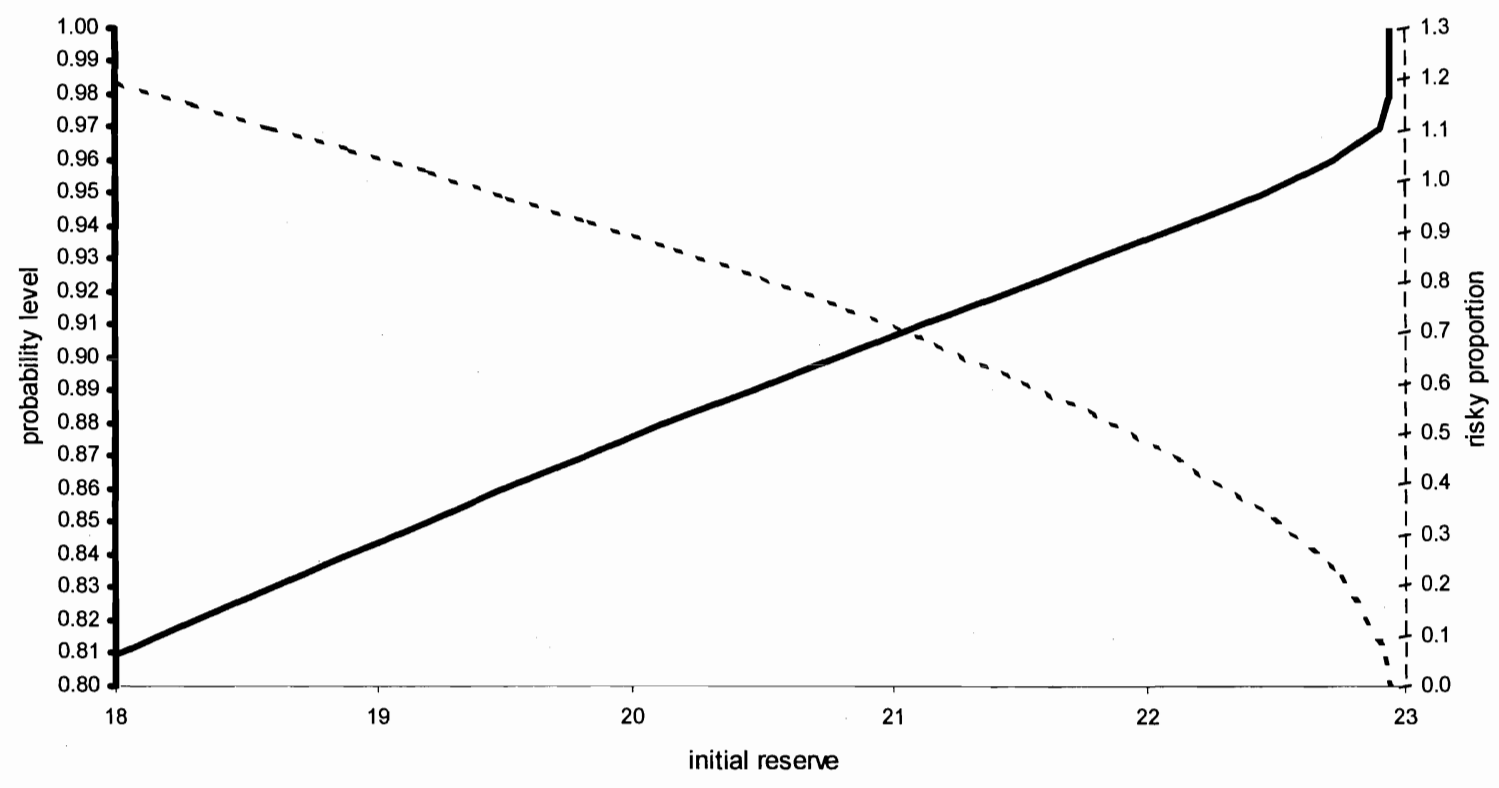

Figure 7: The maximal probability level of reaching the finish $p^{l}$ (solid line - left scale) and the optimal risky proportion $\pi^{l}$ (dotted line - right scale) as a function of the initial reserve $R_{0}$. 
funds. The reserving problem describes situations where funds are set up and invested in order to be able to fulfill a future deterministic consumption pattern. For both problems several optimization criteria were considered.

These portfolio selection problems can be considered as multidimensional in two directions. First, they are multidimensional in terms of time as $n$ yearly returns $Y_{i}(\pi)$ are involved. They are also multidimensional in terms of portfolio choice as the proportions related to the $m+1$ assets have to be chosen.

The classical way to solve the problems we considered in this paper is via Monte Carlo simulation. Take as an example the final wealth problem described in (92). Such a strategy starts with simulating series of outcomes $\left(u_{1}, u_{2}, \ldots, u_{n}\right)$ of the multivariate random vector $\left(U_{1}, U_{2}, \ldots, U_{n}\right)$ with mutually independent marginals that are uniformly distributed on the unit interval. From these simulated values, one determines series of yearly returns $\left(y_{1}(\pi), y_{2}(\pi), \ldots, y_{n}(\pi)\right)$ for each possible portfolio $\pi^{T}=\left(\pi_{1}, \pi_{2}, \cdots, \pi_{m}\right)$. This leads to a simulated value of the quantiles $Q_{1-p}^{+}\left(W_{n}(\pi)\right)$ for all portfolio choices. The optimal investment strategy is then the one that correspond to the largest quantile. Because of the multiple simulations and calculations involved, this method is extremely time-consuming, with a trade-off to be made between speed and accuracy.

In this paper we proposed a way to escape this 'curse of dimensionality'. The comonotonicity approach reduces the time-dimensionality to one dimension, as the randomness of the $n$ dimensional vector of yearly returns is reduced to the randomness of one single uniform variable $U$. Moreover, the comonotonicity technique avoids simulation as analytical expressions for approximations of the quantiles are available. These expressions can be computed very quickly and are highly accurate at the same time. Also the portfolio-dimension is reduced to a single dimension, as we have proven that the optimal (approximate) quantities correspond to portfolios on the Markowitz mean-variance efficient frontier.

Many of the results presented in this paper can be generalized in several directions. A first immediate generalization consists in finding optimal investments mixes when restrictions are set on the proportions held in the different asset classes. E.g. there may be general linear constraints on the weights $\pi_{i}$, such as

$$
l_{j} \leq a_{0}^{j}+\pi^{T} \times\left(\mathbf{a}^{j}-a_{0}^{j} \mathbf{1}\right) \leq u_{j}, \quad j=1, \cdots, c,
$$

with $\mathbf{a}^{j}=\left(a_{1}, a_{2}, \ldots, a_{m}\right)$. These bounds might express restrictions on the individual weights, such as 'the fraction of the portfolio in property must be between $0 \%$ and $15 \%$ ' or 'a fixed percentage of the fund should be available in cash' and 'there shall be no short-selling of assets'. But also more general linear restrictions such as 'the total fraction of the portfolio allocated to all international assets must not exceed $40 \%$ ' can be expressed by a constraint of the form (159). With constraints of this type, the approximations for the investor's optimal portfolio choice of all problems considered in this paper will be found on the adjusted meanvariance efficient frontier $\left\{\left(\sigma\left(\pi^{\mu}\right), \mu\right)\right\}$ where for a given level of the drift $\mu$, the meanvariance efficient portfolio $\pi^{\mu}$ is now defined by

$$
\operatorname{Min}_{\pi \in R} \sigma^{2}(\pi) \text { subject to } \mu(\pi)=\mu,
$$

where $R$ is the set of all portfolios $\pi$ fulfilling the constraints (159). Note however that in this case, the efficient frontier cannot be expressed analytically anymore and the special structure 
of the efficient frontier is destroyed. However, the optimization problem is still quadratic convex and powerful numerical methods are available to solve the mean-variance problem (160).

In this paper, we restricted the optimization function to be a quantile, or a conditional (left) tail expectation. Many results can be generalized to the case where the optimization function is a distortion risk measure. Indeed, as is explained in Dhaene, Vanduffel, Tang, Goovaerts, Kaas \& Vyncke (2004) for instance, any distortion risk measure of a sum of comonotonic random variables such as $W_{n}^{l}(\pi), W_{n}^{c}(\pi), S_{0}^{l}(\pi)$ and $S_{0}^{c}(\pi)$ can be expressed as the sum of the distortion risk measure of the (lognormal) random variables involved.

We considered the portfolio selection problem within the framework of a Black \& Scholes market. In particular we assumed that the drift and volatility of the different asset classes are constant over time and that the yearly returns are lognormally distributed. The results can be generalized in a straightforward way to take into to account the time-dependency of drifts and volatilities. Also many of the results presented here can be generalized to other than normal distributions for the yearly investment returns. In particular, many results can be generalized in a Lévy-type or elliptical-type world. Comonotonic approximations for sums of random variables with distributions of this type are considered in Valdez \& Dhaene (2004) and Albrecher, Dhaene, Goovaerts \& Schoutens (2004).

Acknowledgement 6.1. Jan Dhaene, Marc Goovaerts, Steven Vanduffel and David Vyncke acknowledge the financial support of the Onderzoeksfonds K.U. Leuven (GOA/02: Actuariële, financiële en statistische aspecten van afhankelijkheden in verzekerings- en financiële portefeuilles).

\section{References}

[1] Albrecher, H.; Dhaene, J.; Goovaerts, M.J. Schoutens, W. (2004). "Static hedging of Asian options under Lévy models: the comonotonicity approach", submitted.

[2] Atkinson, D.B.; Dallas, J.W. (2000). Life Insurance : Products and Finance, Society of Actuaries.

[3] Björk, T. (1998). Arbitrage Theory in Continuous Time. Oxford University Press, pp. 311.

[4] Black, F.; Scholes, M. (1973). "The pricing of options and corporate liabilities", Journal of Political Economy, 81 (May-June), 637-659.

[5] Bodie, Z. (1995). "On the risk of stocks in the long run", Financial Analysts Journal, $51(3), 18-22$.

[6] Cesari, R.; Cremonini, D. (2003). "Benchmarking, portfolio insurance and technical analysis: a Monte Carlo comparison of dynamic strategies of asset allocation", Journal of Economic Dynamics and Control, 27, 987-1011.

[7] Denneberg, D. (1994). "Non-additive measure and integral", Kluwer Academic Publishers, Boston. 
[8] Dhaene, J.; Denuit, M.; Goovaerts, M.J.; Kaas, R.; Vyncke, D. (2002a). "The concept of comonotonicity in actuarial science and finance: theory", Insurance: Mathematics $\mathcal{E}$ Economics, vol. 31(1), 3-33.

[9] Dhaene, J.; Denuit, M.; Goovaerts, M.J.; Kaas, R.; Vyncke, D. (2002b). "The concept of comonotonicity in actuarial science and finance: applications", Insurance: Mathematics E Economics, vol. 31(2), 133-161.

[10] Dhaene, J.; Vanduffel, S., Tang, Q.; Goovaerts; M.J.; Kaas, R.; Vyncke, D. (2004). "Solvency capital, risk measures and comonotonicity: a review", www.kuleuven.ac.be/insurance, publications.

[11] Emmer, S.; Klüppelberg, C.; Korn, R. (2001). "Optimal portfolios with bounded capital at risk", Mathematical Finance, Vol. 11 (4), 365-384.

[12] Jagannathan, R.; Kocherlakota, N. (1996). "Why should older people invest less in stocks than younger people?", Federal Reserve Bank of Minneapolis Quarterly Review, 20(3), 11-23.

[13] Kaas, R.; Goovaerts, M.J., Dhaene, J.; Denuit, M. (2001). "Modern Actuarial Risk Theory", Kluwer Academic Publishers, pp. 328.

[14] Li, Z.F.; Ng, K.W.; Tan, K.S.; Yang, H. (2003). "A closed-form solution to a dynamic portfolio optimization problem", forthcoming.

[15] Markowitz, H. (1952). "Portfolio selection", Journal of Finance, 7, 77-91.

[16] Markowitz, H. (1959). Portfolio Selection - Efficient Diversification of Investments, Wiley, New York.

[17] Marshall, J.F. (1994). "The role of the investment horizon in optimal portfolio sequencing"; Financial Review, 29(4), 557-576.

[18] Merton, R. (1971). "Optimum consumption and portfolio rules in a continuous-time model", Journal of Economic Theory, 3, 373-413.

[19] Merton, R. (1990). Continuous Time Finance, Cambridge, Blackwell.

[20] Milevsky, M.; Ho, K.; Robinson, C. (1997). "Asset allocation via the conditional first exit time or how to avoid outliving your money", Review of Quantitative Finance and Accounting, 9(1), 53-70.

[21] Milevsky, M.; Posner, S. (1998). "Asian options, the sum of lognormals and the reciprocal Gamma distribution", Journal of Financial and Quantitative Analysis, vol. 33, September 1998, 409-422.

[22] Milevsky, M.; Robinson, C. (2000). "Self-annuization and ruin in retirement, with discussion", North American Actuarial Journal, 4(4), 112-129. 
[23] Milevsky, M. (2003). "Space-time diversification: Which dimension is better?", Journal of Risk, vol. 5(2), to be published.

[24] Müller, A. (1997) Stop-loss order for portfolios of dependent risks. Insurance Math. Econom. vol.21(3), 219-223.

[25] Olivieri, A.; Pitacco, E. (2003). "Solvency requirements for pension annuities", Journal of Pension Economics and Finance, vol. 2(2), 127-157.

[26] Rubinstein, M. (1991). "Continuously rebalanced investment strategies", The Journal of Portfolio Management, Fall, 78-81.

[27] Roy, A. (1952). "Safety first and the holding of assets", Econometrica, 20, 431-449.

[28] Samuelson, P.A. (1989). "The judgement of economic science on rational portfolio management: Timing and long horizon effects", The Journal of Portfolio Management, $16(1), 4-12$.

[29] Steinbach (2001). Markowitz revisited: mean-variance models in financial portfolio analysis", SIAM Review, 43(1), 31-85.

[30] Valdez, E.; Dhaene, J. (2004). "Bounds for sums of dependent log-elliptical risks", forthcoming.

[31] Vanduffel, S.; Dhaene, J.; Goovaerts, M.; Kaas, R.(2003). "The hurdle-race problem", Insurance: Mathematics \& Economics 33, 405-413.

[32] Von Neumann, J; Morgenstern, O. (1947). "Theory of games and economic behavior", Princeton University Press, Princeton.

[33] Yaari, M.E. (1987). "The dual theory of choice under risk", Econometrica 55, 95-115.

[34] Young, V.R. (2004). "Optimal investment strategy to minimize the probability of lifetime ruin", to be published. 